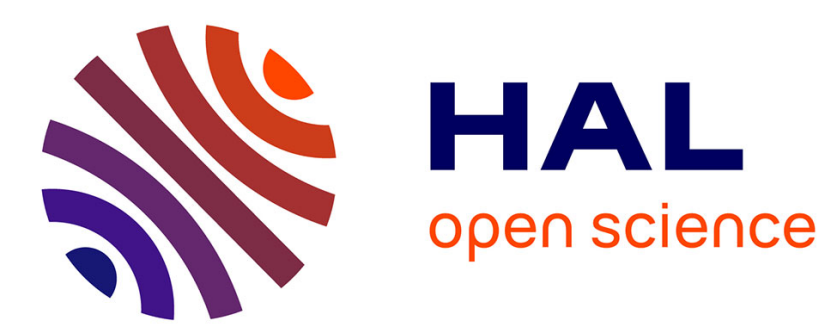

\title{
Ontology matching benchmarks: generation, stability, and discriminability
}

Jérôme Euzenat, Maria Rosoiu, Cassia Trojahn dos Santos

\section{To cite this version:}

Jérôme Euzenat, Maria Rosoiu, Cassia Trojahn dos Santos. Ontology matching benchmarks: generation, stability, and discriminability. Journal of Web Semantics, 2013, 21, pp.30-48. 10.1016/j.websem.2013.05.002 . hal-01123792v2

\section{HAL Id: hal-01123792 \\ https://hal.inria.fr/hal-01123792v2}

Submitted on 12 Dec 2013

HAL is a multi-disciplinary open access archive for the deposit and dissemination of scientific research documents, whether they are published or not. The documents may come from teaching and research institutions in France or abroad, or from public or private research centers.
L'archive ouverte pluridisciplinaire HAL, est destinée au dépôt et à la diffusion de documents scientifiques de niveau recherche, publiés ou non, émanant des établissements d'enseignement et de recherche français ou étrangers, des laboratoires publics ou privés. 


\title{
Ontology matching benchmarks: generation, stability, and discriminability
}

\author{
Jérôme Euzenat ${ }^{\mathrm{a}, *}$, Maria-Elena Roşoiu ${ }^{\mathrm{a}}$, Cássia Trojahn ${ }^{\mathrm{b}, * *}$ \\ ${ }^{a}$ INRIA \& $L I G$, Grenoble, France \\ ${ }^{b}$ IRIT \& UTM2, Toulouse, France
}

\begin{abstract}
The OAEI Benchmark test set has been used for many years as a main reference to evaluate and compare ontology matching systems. However, this test set has barely varied since 2004 and has become a relatively easy task for matchers. In this paper, we present the design of a flexible test generator based on an extensible set of alterators which may be used programmatically for generating different test sets from different seed ontologies and different alteration modalities. It has been used for reproducing Benchmark both with the original seed ontology and with other ontologies. This highlights the remarkable stability of results over different generations and the preservation of difficulty across seed ontologies, as well as a systematic bias towards the initial Benchmark test set and the inability of such tests to identify an overall winning matcher. These were exactly the properties for which Benchmark had been designed. Furthermore, the generator has been used for providing new test sets aiming at increasing the difficulty and discriminability of Benchmark. Although difficulty may be easily increased with the generator, attempts to increase discriminability proved unfruitful. However, efforts towards this goal raise questions about the very nature of discriminability.
\end{abstract}

Keywords: Ontology matching, Matching evaluation, Test generation, Semantic web.

\section{Introduction}

Heterogeneity is inherent to open environments like the semantic web. Thus, ontology matching is very important to overcome ontology heterogeneity and many systems have been proposed for this purpose [10]. However, matching ontologies is not a deductive task for which it is possible to check if a matcher is compliant with a specification, like for SPARQL querying or OWL reasoning. Hence, evaluation of ontology matchers is of paramount importance.

Evaluating ontology matching systems may be achieved in different ways. The most common one consists of providing matchers with two ontologies and comparing the returned alignment with a reference alignment [9]. However, this raises the issue of the choice of ontologies and the validity of the reference alignments.

Since 2004, the Ontology Alignment Evaluation Initiative (OAEI) ${ }^{1}$ makes available a collection of test sets for evaluating matching systems. One such test set is Benchmark (we use the term Benchmark with a capital B for referring to this test set). It is a well-defined set of tests in which each test is composed of two ontologies and a reference alignment. The tests are based on one particular ontology, from the bibliographic domain, and systematic alterations of this ontology, e.g., removing classes, renaming properties.

\footnotetext{
This paper improves over [18], in particular by providing results based on more extensive test bases and in generating difficult and discriminant test sets. It has been thoroughly revised.

${ }^{*}$ Corresponding author

** Work performed when this author was with INRIA.

Email addresses: Jerome.Euzenat@inria.fr (Jérôme Euzenat), rosoiu.maria@gmail.com (Maria-Elena Roşoiu), Cassia.Trojahn@irit.fr (Cássia Trojahn)

${ }^{1}$ http://oaei.ontologymatching.org/
} 
The Benchmark test set was designed with the aim of covering the various situations in which a matcher may be (called problem space). However, this test set can be criticised on three main aspects: (a) lack of realism: tests are mechanically generated, (b) lack of variability: it always uses the same seed ontology altered in the exact same way, and (c) lack of discriminability: the tests are not difficult enough to discriminate well matchers. While the drawback (a) has been overcome by other test sets made available by OAEI, in this paper we are particularly interested in investigating (b) and (c).

To that extent, we have developed a test generator that may be used with any seed ontology and that allows for fine tuning the input parameters, as well as randomised modifications over the ontology entities. The generator framework is extensible - it is possible to add new alteration capabilities - and flexible - it can be used through a clearly defined API to generate various kinds of tests.

This generator enables us to evaluate the relevance of the Benchmark test set: by reproducing this test set and using it to evaluate different matchers, under the same conditions, we can assess how much the obtained results were dependent on the particular seed ontology or on the particular matcher.

We ran a set of matchers on the generated tests, which sheds light on the results obtained so far with Benchmark. Concerning Benchmark, we establish that:

- Results obtained by different generations of Benchmark are very stable, i.e., their standard deviation is less than 1 percentage point on average.

- The difficulties encountered by a particular matcher at a test are preserved across seed ontologies, even if the difficulty of each test set is different.

- A bias towards the original Benchmark and its domain can be observed.

- It is not possible to identify a general order between matchers consistent across seed ontologies.

These points support the initial design goals of Benchmark: to be able to identify strengths of matchers over the whole problem space and not to find which matcher is "the best one". The last point may however be related to the lack of discriminability of the test set: matchers obtain very close scores.

Furthermore, we have extended the framework in order to generate tests of increasing difficulty and discriminability. The test generator can easily generate test sets of increasing difficulty. However, such tests do not provide increased discrimination. Increasing discrimination has proved challenging. This may be due to two main factors: matchers may be very similar, and discriminability is not precisely defined. We use the test generator to discuss and illustrate these issues.

In summary, as main contributions, this paper:

- provides a very versatile benchmark generator (§3),

- uses this generator to measure meaningful properties of ontology matchers and to assess the validity of Benchmark principles and its stability $(\S 4)$,

- shows how new tests may be generated on the same model for evaluating scalability, difficulty and discriminability $(\S 5)$.

The remainder of the paper is structured as follows. In Section 2, we present the state-of-the-art in ontology matching test generation. Section 3 presents the architecture of our test generator and the strategy to reproduce the Benchmark test set. In Section 4, we experimentally establish properties of Benchmark using new generated test sets. Section 5 investigates different ways to use the test generator in order to generate more difficult and discriminant benchmarks. Finally, conclusions and future work are presented in Section 6.

\section{Ontology matching evaluation and test generation}

In this section, we briefly present the current setting of ontology matching evaluation (§2.1), the Benchmark test set $(\S 2.2)$ and its limitations $(\S 2.3)$. We survey the state-of-the-art in alignment test generators $(\S 2.4)$. The interested reader can find a broader overview of ontology matching evaluation in [9]. 


\subsection{Evaluating ontology matching systems}

Ontology matching [10] can be seen as an operation that takes as input two ontologies $\left(o\right.$ and $\left.o^{\prime}\right)$, a set of parameters, a possibly empty partial alignment $\left(A^{\prime}\right)$ and a set of resources, and outputs an alignment $(A)$ between these ontologies (Figure 1, dotted lines). A resource may be a particular lexicon used for matching terms or an ontology that may be used as an intermediate ontology between the ontologies to be matched. Parameters may be different weights put on features of the ontologies, e.g., labels, structure, instances, or switches indicating which part of the program may be activated, e.g., for fast computation or for emphasising recall.

In this paper, a matcher is a particular program performing the ontology matching operation together with its resources and parameters, so it is always the exact same piece of program run under the same conditions.

An alignment can be defined as a set of correspondences. A correspondence between two ontologies $o$ and $o^{\prime}$ is a triple $\left\langle e, r, e^{\prime}\right\rangle$, such that $e$ is an entity belonging to the first ontology, $e^{\prime}$ is an entity belonging to the second ontology, and $r$ is a relation, e.g., equivalence or subsumption, between them.

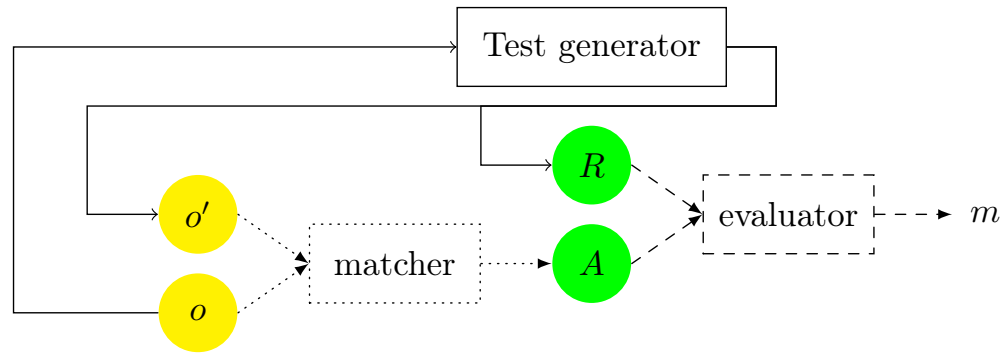

Figure 1: Test generation (plain lines), ontology matching (dotted) and evaluation (dashed). The test generator provides two ontologies $\left(o\right.$ and $\left.o^{\prime}\right)$ and a reference alignment $(R)$. The matcher outputs an alignment $(A)$ that the evaluator compares to the reference alignment in order to compute a measure $(m)$.

A matcher can be evaluated by comparing its output alignment $(A)$ with a reference alignment $(R)$ using some measure (Figure 1, dashed lines). Typical measures are precision, recall and F-measure [9]. Thus, in order to evaluate a matching system, one has to generate test sets in which a test is composed of two ontologies to be matched $\left(o\right.$ and $\left.o^{\prime}\right)$ and a reference alignment $(R)$. The test generation considered here starts with a seed ontology $(o)$ for which the test generator provides both an altered ontology $\left(o^{\prime}\right)$ and the corresponding reference alignment.

In order to abstract from actual ontology matching problems, we distinguish between the problem space, the solution space and the alteration space. The problem space corresponds to the space of various manifestations of ontology heterogeneity which require matching ontologies. The solution space defines the space of ontology matching problems that can be solved by matchers. The alteration space is a space of ontology matching problems that may be obtained by altering ontologies (as in Figure 2). We use the term space because these situations are not unrelated and their proximity is expected to be meaningful. This is illustrated in $\S 3.4$.

\subsection{The Benchmark test set}

The Benchmark test set aims at assessing the strengths and the weaknesses of matching systems, depending on the availability of ontology features, i.e., the availability of instances, properties or labels in the ontology. This test set has 111 tests, requiring to match an ontology written in OWL-DL with another one:

- Tests 1xx - compare the original ontology with itself, a random one and its generalisation in OWL-Lite.

- Tests $2 \mathrm{xx}$ - compare the original ontology with the ontology obtained by applying the following set of modifications to it (Figure 2): 


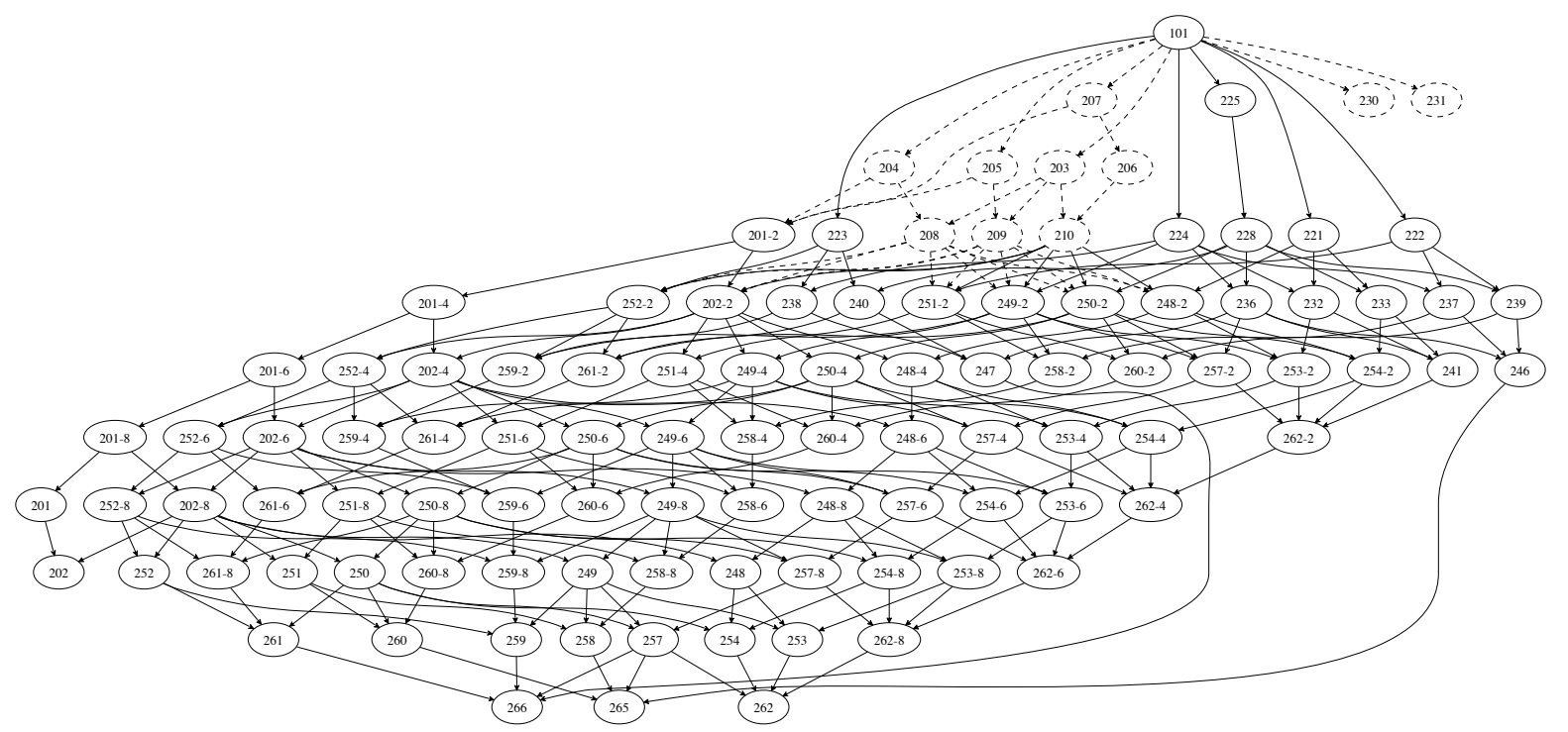

Figure 2: The Benchmark semi-lattice - the higher the test in the hierarchy, the easier it is. Dashed lined tests were not generated in the experiments reported here (see $\S 4$ ).

- names (naming conventions: synonyms, random strings, different generalisations, translation into other language),

- comments (no comment),

- hierarchy (flattened hierarchy / expanded hierarchy / no specialisation),

- instances (no instance),

- properties (no property, no restriction),

- classes (flattened classes / expanded classes).

- Test 3xx - compare the original ontology with real ones found on the web.

Since 2004, Benchmark has been generated from the same seed ontology through the same set of XSLT stylesheets. This means, in particular, that no random modification is applied to these ontologies: the same $20 \%$ of classes are renamed and this renaming is always the same, i.e., "editor" is always renamed as "dzajj". This has advantages for studying the evolution of the field, because the test is strictly the same, but it has drawbacks as well, because the alterations are known in advance by evaluation participants.

\subsection{Benchmark limitations}

The Benchmark test set can be criticised on three main aspects:

Lack of realism Benchmark is not realistic because it covers a whole systematic alteration space and, in reality, a matcher is not faced with such a space.

Lack in variability Benchmark is always the same test set, hence it is not variable. This covers three slightly different kinds of problems: (a) it can only be used with one seed ontology, (b) it always applies the same transformations (to the same entities), instead of applying them randomly, and (c) it is not flexible in the sense that it is not possible to produce an arbitrary test (such as $12 \%$ renaming, $64 \%$ discarding properties).

Lack of discriminability Benchmark seems, in general, easy enough to OAEI participants so that it does not really allow them to make progress and it is not discriminant enough to compare them [13]. This is because many of the proposed tests are easy and only a few of them are really difficult. 
Our goal is to address variability and discriminability by producing a test generator (a) independent from the seed ontology, (b) with random modifications, and (c) which allows for fine tuning parameters in order to cover the alteration space with any precision. With such a test generator, it is also possible to generate different tests than Benchmark, focusing on particular application profiles or particularly difficult cases.

We do not address the lack of realism because Benchmark has been designed to cover the problem space and not to offer one realistic profile. One may argue that we currently consider an alteration space, instead of a problem space, and that the term "problem space" assumes some realism, i.e., that these problems actually occur. This is right, but this alteration space is our attempt to cover the problem space, and not to represent the problem space itself. Other initiatives, such as other tracks of OAEI and other generators, address the realism issue $[2,9]$.

Some general purpose requirements for semantic web knowledge-based system benchmarks are scalability, openness to new participants, platform independence, meaningful metrics, and reproducibility [14]. These criteria are those which are targeted by a new Benchmark generation: having a variable and flexible test generator allows for scalability and reproducibility (as will be shown in §4); using standardised input and output formats ensures openness; platform independence is the goal of both the test generator and the SEALS platform ${ }^{2}$; finally, we think that $\S 4$ shows that Benchmark indeed measures permanent and meaningful properties of matchers.

Moreover, five properties were found essential (though not exhaustive, but good enough) for ontology matching evaluation test sets [13]. These are: complexity: that it is hard for state-of-the-art matching systems; incrementality: that it is effective in revealing weaknesses of the state-of-the-art matching systems; discriminability: that it discriminates sufficiently among the various matching solutions; monotonicity: that the matching quality measures calculated on the subsets of the test set do not differ substantially from the measures calculated on the whole test set; and correctness: that it can be considered as a correct tool to support the improvement of matching solutions.

Of those, complexity, incrementality and discriminability resort to what we identified as lack of difficulty and discriminability. Later $(\S 5)$, we will consider both discriminability and difficulty in an incremental way. Monotony is not sufficiently precisely defined for qualifying. Obviously, if a test set like Benchmark offers various configurations, it will not be monotonous with any subset. However, we will show in $\S 4$ that Benchmark is indeed monotonous in different ways. Finally, under this informal definition of correctness, there are good reasons to consider Benchmark as "correct" (it is widely used by matcher developers, participating or not to OAEI).

Three different properties have also been considered [2]: consistency: each test should evaluate a particular feature; completeness: there is a test for any (important) feature; and minimality: there is no more than one test per feature. As soon as a combination of alterations can be considered a feature, Benchmark can be considered as following these rules.

\subsection{Related work}

Many efforts took inspiration from the original OAEI Benchmark in generating test sets through alteration. Both IIMB [11] and ONTOBI [25] benchmarks have been developed for testing instance-based ontology matching systems. On the basis of Benckmark-like tests, they add a large amount of instances based on external sources (the internet movie database or wikipedia data). These efforts publish the resulting test set and not the generator.

The Swing approach [12], based on the experience of IIMB, is a further effort to generate instance matching test sets. Swing classically distinguishes between the TBox, i.e., the vocabulary, and the ABox, i.e., the data. It works in two steps: a data acquisition step collects data to be matched and induces a TBox from this data. It then adds expressiveness to this TBox both automatically and manually. In the test generation step, from that ontology which remains the same over all tests, different ABoxes (test cases)

\footnotetext{
${ }^{2}$ http://seals-project.eu
} 
are generated by altering data values, e.g., token addition, name style abbreviation, changing data format or number format, and properties, e.g., adding or suppressing properties, changing property depth.

In XML schema matching, STBenchmark [1] offers a schema generator (SGen) which generates tests (pairs of schemas) by altering a source schema based on the specification of 11 scenarios (base alterators). It does not generate any reference results. The scenarios are defined through a set of input parameters which include the characteristic parameter, e.g., nesting depth, number of sub-elements, length of joint paths, the standard deviations to be applied for sampling each characteristic, and a repetition parameter. Schema instances can also be included in the generated schemas. Like Benchmark, STBenchmark uses XSLT.

XBenchMatch [7] provides a way for benchmarking schema matching systems, but is not a test generator. However, it provides a testbed involving a large schema corpus that can be used to benchmark new schema matching algorithms. It aims at providing a systematic way for analysing if a schema matching tool is appropriate in a given context. XBenchMatch takes as input sets of correspondences or integrated XML schemas produced by matchers and applies a set of metrics for evaluating the quality of the input and performance of the matching tool, for instance. XBenchMatch can be extended programmatically.

Another automatic generator inspired by Benchmark [3] takes as seed ontology a random tree which is computed using a Gaussian distribution of the number of children per node. The second ontology is obtained from the first one by applying a set of alterations, similar to the ones used in Benchmark, such as label replacement, word addition or removal in labels, node deletion and node child addition and children shuffling. Then, these two generated ontologies are used to generate alignments between them. The aim of generating the original ontology is to perform realistic tests and to allow a wider coverage of variations in their structure. The same technique has been used for testing web service matching in the geographical domain [22].

An automatic generator aiming at realism has been proposed in [20]. This generator satisfies two requirements: (a) to generate the structure and the instances of two taxonomies, and (b) to generate an alignment between these two generated taxonomies. Both taxonomies must have a fixed size and a Boltzmann sampler is used to achieve this. The probabilistic model used ensures an equal probability of appearance of a tree having a given size. Therefore, the input data is controlled using this sampler. The number of child nodes is controlled as well. Then, the alignment between the two taxonomies is generated in a way that does not contradict the generated data. To achieve this goal, three constraints were enforced: the alignment must not introduce a cycle in the newly obtained graph (the alignment and the two given taxonomies), the alignment must not contradict the knowledge of the two taxonomies and they must not entail each other.

For directory matching, the TaxMe test set [13] is built from existing web directories with the aim of providing large scale and realistic test sets for matching evaluation. However, this test set only contains a subset of the reference alignment and the generation mechanism matches "concepts" which are indexing documents in common. It is thus comparable to a matching technique and thus prone to bias towards similar techniques.

Table 1 compares the different work. Some of them have not been made available as generators (ONTOBI and TaxMe); some are dedicated to XML (or database) schemas (STBenchmark and XBenchMatch). Swing is dedicated to the alteration of the ABox part of the ontologies. In fact, the approach developed here is complementary to that of Swing and could take advantage of its ABox alterators. The most suitable systems for ontology matching test generation are the systems described in [3] and [20] which were not available to reuse and offer little flexibility.

In conclusion, none of the available generators is both able to generate OAEI Benchmark and go beyond it by offering the flexibility that the original Benchmark lacked as discussed in $§ 2.3$. Hence, we developed the test generator that is described hereafter.

\section{A modular benchmark test generator}

The OAEI Benchmark test set suffers from lack of variability and discriminability. These problems affect the relevance of Benchmark as a good basis for comparing matchers, but not for matcher developers themselves. In order to overcome these problems, we developed a test generator in Java, based on the Jena 


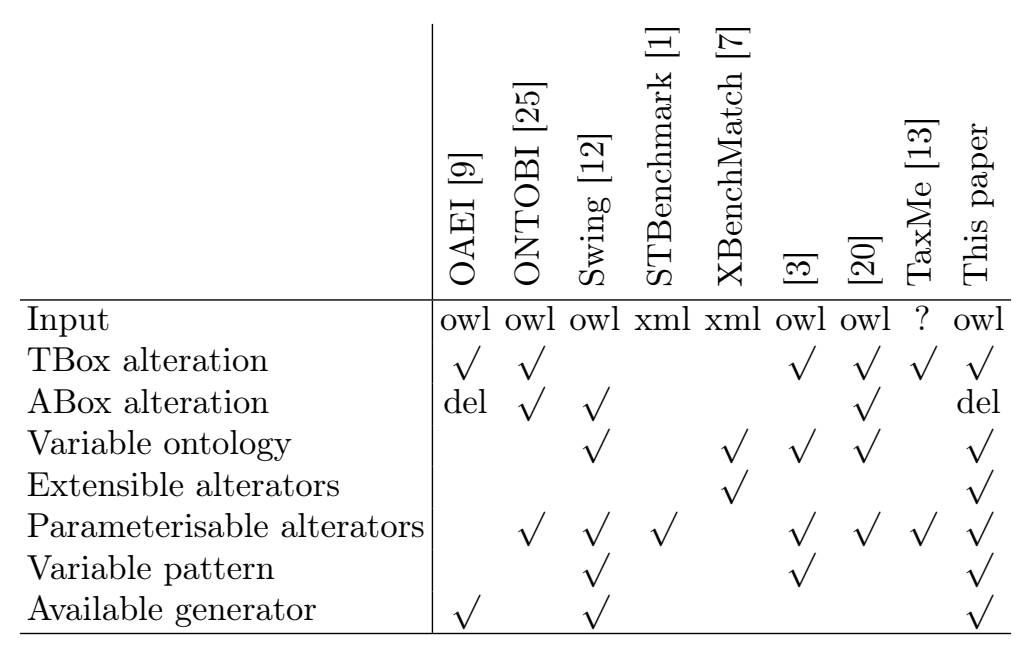

Table 1: Comparison of the different test generators.

$\mathrm{API}^{3}$ and integrated within the Alignment $\mathrm{API}^{4}$. We present the principles of the generator (§3.1) and illustrate them through an example of generation (§3.2). We present preliminary tests (§3.3) and show how generating a test set allows for characterising matchers $(\S 3.4)$.

\subsection{Generator principles}

The main principle of the test generator is that, from one ontology, it can generate an altered one. The input ontology may be a regular ontology or an artificially generated one: this may be useful for generating scalability tests.

Because the alteration is known, if an input alignment is provided it can be altered in the same exact way, providing a new reference alignment. If no input alignment is given, an implicit alignment between all named entities of the input ontologies is assumed.

We designed the Alterator interface taking as input an ontology and an alignment between this ontology and the seed ontology. This module outputs an altered ontology and its alignment with the seed ontology (Figure 3). This allows for applying alterations in sequence and retrieve a test at each step.

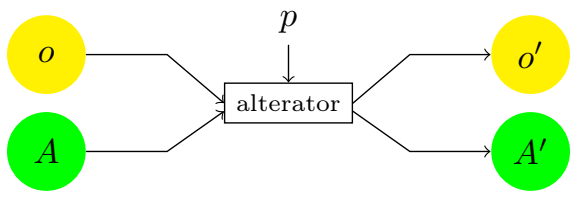

Figure 3: Modular structure of test generators (Alterator).

Thus, extending the test generator is easy since it is sufficient to implement new alterators and to declare them to the AlteratorFactory.

Elementary alterators. Elementary alterators are implementation of the Alterator interface that apply one particular alteration to an ontology. In order to assess the capability of matchers with respect to particular ontology features, we consider the following alterations: remove percentage of classes; remove percentage of properties; remove percentage of comments; remove percentage of restrictions; remove all

\footnotetext{
${ }^{3}$ http://jena.sourceforge.net/ontology/index.html

${ }^{4}$ http://alignapi.gforge.inria.fr
} 
classes from a level; rename percentage of classes; rename percentage of properties; add percentage of classes; add percentage of properties; add a number of classes to a specific level; flatten a level; remove individuals. A detailed description of elementary alterators is provided in Appendix C.

Alterators may be composed and manipulated programmatically, so as to dynamically adapt the degree of alteration for instance.

Generating a test case. To modify an ontology according to a set of parameters we have defined TestGenerator as illustrated in Figure 4. It receives as input the seed ontology and the parameters which represent the alterations to be applied. The output is the modified ontology and the reference alignment. The implementation of the TestGenerator composes Alterators in a serial manner. This can produce an arbitrary test.

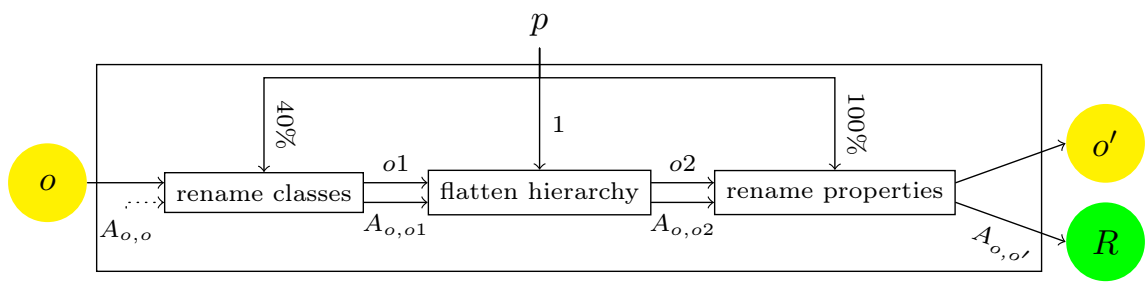

Figure 4: One-shot test generation by composing alterators (TestGenerator). Subscripts on the arrows illustrate the evolution of the alignment which always refers to the input ontology $(o)$.

Generating a test set. The test generator framework can also be used to reproduce test sets such as Benchmark. For that purpose, we designed the TestSet abstraction, in which test sets are described as applications of TestGenerator. The program will either generate all the required tests independently, by running TestGenerator each time from the beginning (Figure 5), or generate them sequentially, as the initial Benchmark test set, by using a previous test and altering it further (Figure 6). In the latter case, this corresponds to selecting paths in the semi-lattice of Figure 2 which cover the whole test set.

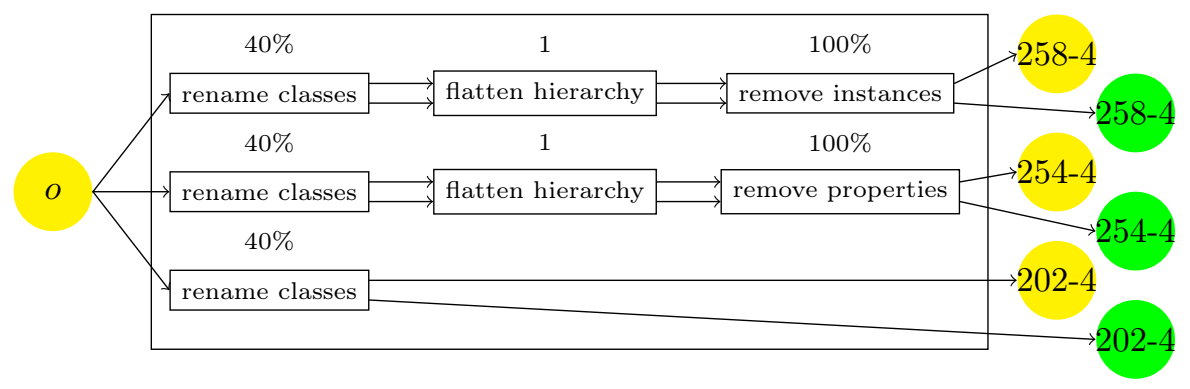

Figure 5: Random test set generation by combining test generators (TestSet).

The proposed approach is extensible because it allows for implementing new alterators as very simple components that can be exploited in generating test sets. It is also flexible, as it can be used to generate complete test sets covering the whole alteration space with a varying degree of precision (incrementing the alteration proportion by $50 \%$ or by $2 \%$ ), or more specific test sets aiming at identifying specific problems (see $\S 5)$.

\subsection{Example of test case generation}

As an example, consider the generation of test \#258-4 from the classic Bibliography ontology. Figure 7 presents a simplified view of the different generated ontologies and alignments. This test renames $40 \%$ of the classes, suppresses one class level and suppresses all instances. For this purpose, it uses four different alterators which, at each step, generate a well-identified test: 


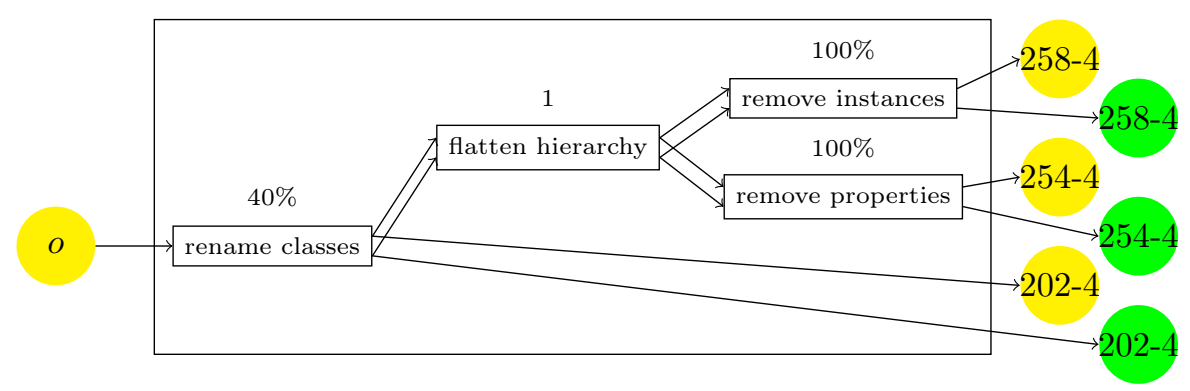

Figure 6: Continuous test set generation (forcing monotony) (TestSet).

Empty does not modify the ontology but generates an alignment expressing all equivalences between named entities of the ontologies. This outputs test \#101 (see §2.2).

Rename classes, provided with a parameter of $40 \%$, will randomly rename that amount of entities, in Figure 7, InProceedings is renamed into Culbrdeo and creator is renamed into ovxylty. This may affect individuals because they are described through properties and classes. The alignment is altered in the same way by renaming the same entity URIs. This outputs test \#202-4.

Flatten level, with level 1 as parameter, will suppress all classes at level 1 in the hierarchy. All constraints applying to these classes will be attached to their subclasses (which will be attached to their superclasses) and all individuals attached to these classes will remain attached to their superclasses. Correspondences involving the suppressed entities are suppressed from the alignment. This outputs test \#251-4.

Remove instances, with parameter $100 \%$, will simply suppress all individuals from the ontology. Since the individuals are not present in the alignment, nothing is changed in it (except using the URIs of the newly produced ontology). This outputs test \#258-4.

\subsection{Preliminary observations}

Before evaluating matchers in the large, we proceeded with a preliminary evaluation of matchers. We report some of the observation from this first use of the test generator, because they may be useful to others. We first noted that matcher behaviour was often not monotonic with respect to the expected difficulty of tests. This did not occur in the original Benchmark because tests were generated from one another in increasing difficulty order. We validated this interpretation by adding the opportunity to generate more continuous test sets (§3.3.1). We also noticed that randomly generated tests may have variable results depending on the order in which alterators were applied and we adopted a suitable generation order (§3.3.2).

\subsubsection{Random vs. continuous policies}

Contrary to expected, matchers did not show a continuous degradation of their performances as more alterations were applied: it may happen that a matcher had better results at a particular test than at a less altered one. This may be caused by two different sources: (i) Matchers have a non deterministic behaviour, i.e., they use randomisation methods as do systems based on evolutionary computation. Such matchers usually find a local optimum depending on the initial alignment. So they can, by chance, return a better result to a more difficult test. Other matchers analyse the ontologies to match and run different matching components depending on their characteristics. They may have a non homogeneous behaviour because the generated ontologies have different characteristics. Finally, other matchers use resources on the web changing over time, such as search engines, so that the results are dependent of the query context. (ii) Because tests are generated independently from each others (Figure 5), it may happen by chance that a test with more alteration is easier than a test with less alteration. In the former case, matcher developers 


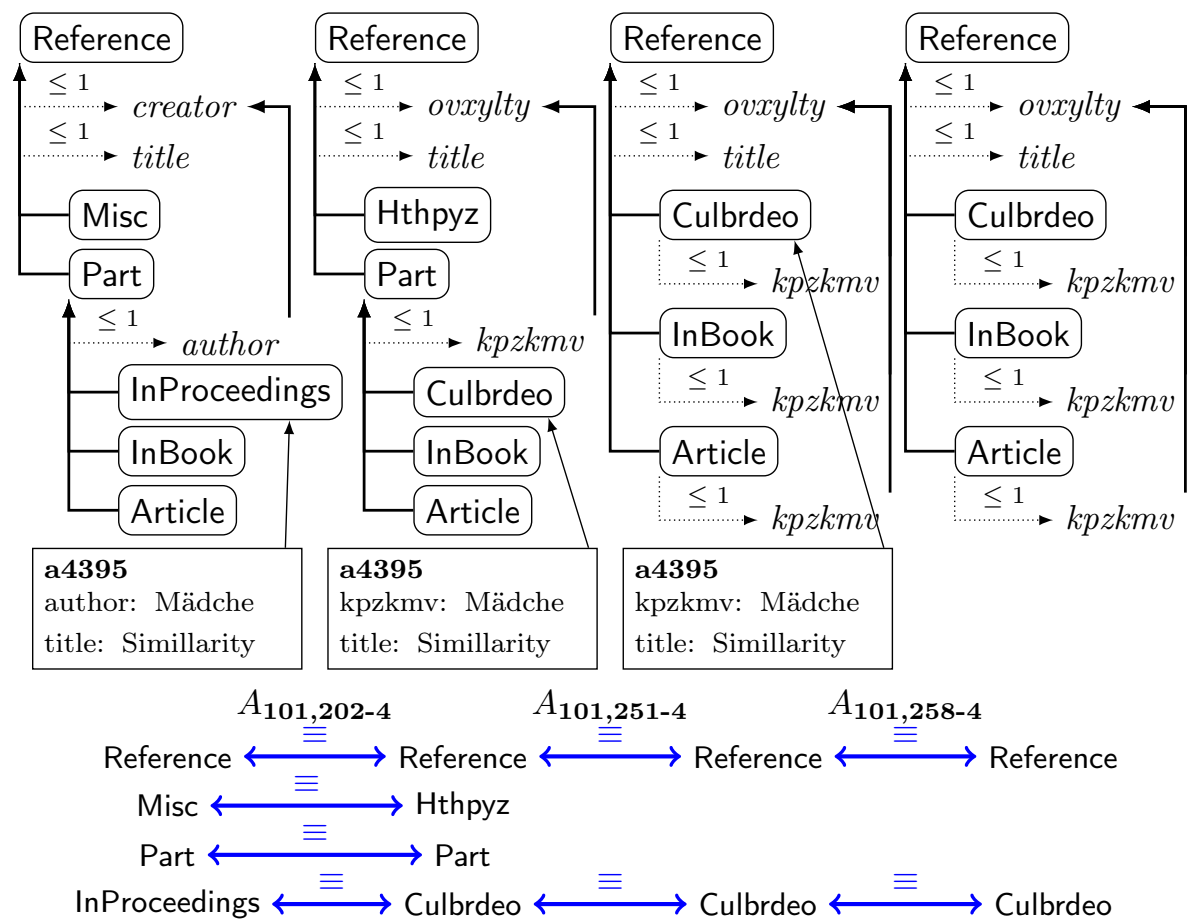

Figure 7: Test generation example (classes are in rounded boxes, properties are in italics, instances in rectangular boxes). The upper part shows the evolution of the ontology; the lower part that of the alignment (always between the 101 ontology and the target ontology).

are used to the behaviour of their systems, but the latter case will affect all matchers and puzzle matcher developers.

This behaviour is only observable locally, i.e., on one test set, and only for a few matchers. When averaging several test set results, matcher behaviours are, on average, continuous. However, this made difficult for tool developers to read one Benchmark test result.

We countered this effect by generating continuous tests (Figure 6) the same way as the initial Benchmark was generated. In this case, new tests are generated from previous ones with the modular architecture of the generator. However, we also observed that the averaged results are the same with either random or continuous generation (see $\$ 4.3$ ). In the results reported below, unless stated otherwise, the results are generated randomly.

\subsubsection{Modification dependencies}

We observed that test difficulty may not be the same across tests supposed to have the same amount of alteration. This is explained by the dependency between alterations. For instance, if one wants to remove $60 \%$ of classes and to rename $20 \%$ of classes, three extreme cases may happen (as illustrated in Figure 8):

- rename $20 \%$ of classes and then remove $60 \%$ of classes, including all renamed classes. In this situation, the test is easier than expected because all renamed classes have been removed;

- rename $20 \%$ of classes and then remove $60 \%$ of classes, including a part of renamed classes. In this situation, the test is as hard as expected because the required proportion of the renamed classes has been removed;

- rename $20 \%$ of classes and then remove $60 \%$ of classes, without removing any of the renamed classes. In this situation, the test is harder than expected because none of the renamed classes has been removed. 


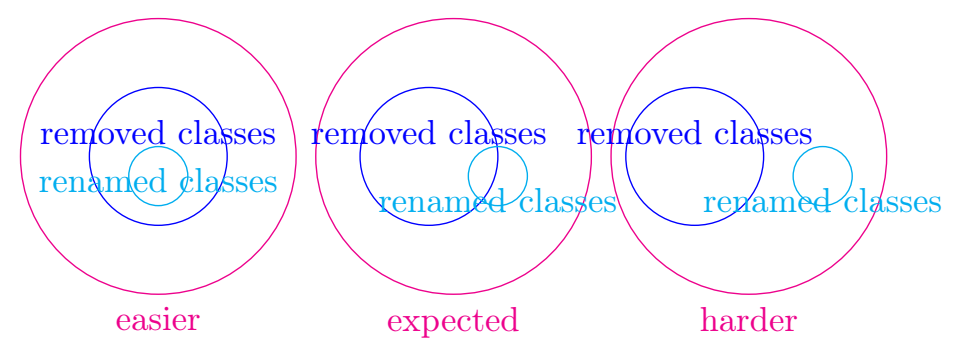

Figure 8: Test dependency: depending on which entities are affected by changes, the test difficulty may vary.

Hence, a random disposition of parameters might reduce the really hard cases. As can be seen from the example, the nominal expected case may be restored by removing $60 \%$ of the classes before renaming $20 \%$ of the remaining ones. Therefore, we established a relevant order for parameters: remove classes, remove properties, remove comments, remove restrictions, add classes, add properties, rename classes, rename properties. In this way, we obtained the expected results. This order helps determining the paths in Figure 2 used for generating Benchmark. Such an order was not previously observed in the Benchmark test set because the value of parameters, but rename resources, was always $100 \%$.

\subsection{Characterising matchers}

Benchmark has been created for characterising matchers, i.e., indicating in which situation they perform adequately. This may, of course, be obtained by running the test corresponding to the actual situation, e.g., \#258-4, and compare the results of matchers. This may also be obtained by considering a more detailed view of the whole Benchmark, instead of an aggregated figure such as average F-measure. Indeed, the more natural way of doing it would be to display Figure 2 with an indication, e.g., colour, of matcher performances. This would be still uneasy to understand because there are many tests and their horizontal ordering is quite random.

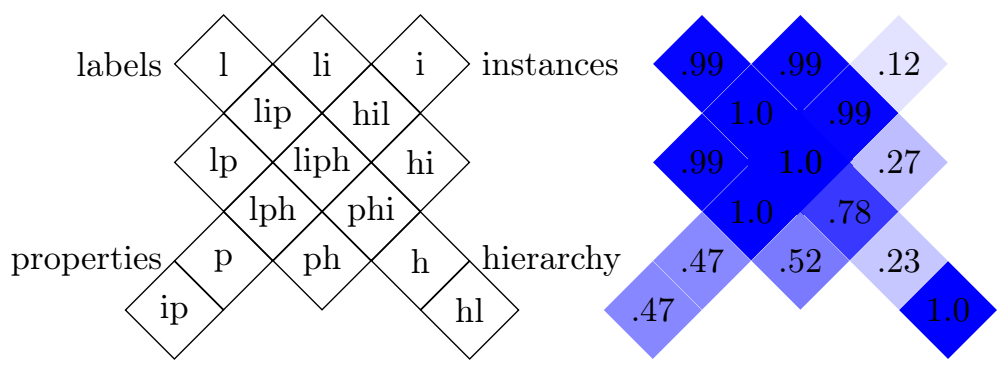

Figure 9: Matcher performance display on a grid preserving as much as possible the topology of tests (left). Each cell corresponds to the availability of some features in the test $(l=$ labels and comments, $p=$ properties, $\mathrm{i}=$ instances, $\mathrm{h}=$ hierarchy). A sample display of $\mathrm{F}$-measure (the darkest the best) generated by GroupOutput of the Alignment API (right).

Figure 9 provides a display that synthesises the obtained results by aggregating in one cell a group of tests in which the ontologies to compare share a common set of characteristics, namely, that the same set of features has been altered [8]. Each cell is presented with a colour representing the average of the F-measure in each of these tests. The darker the cell, the better the algorithm. These diagrams are topologically correct, i.e., if a cell touches another cell on one side, they differ from only one "feature", but not complete, i.e., it is not possible to present all these connections in a planar way.

Such diagrams may be obtained for any measure that can be rendered in colour: we use them with F-measures here. 
Their use for an application developer consists of characterising the cell in the diagram which corresponds to the application data (by the presence/absence of labels, properties, instances or hierarchy) and to select the best matcher with regard to this cell.

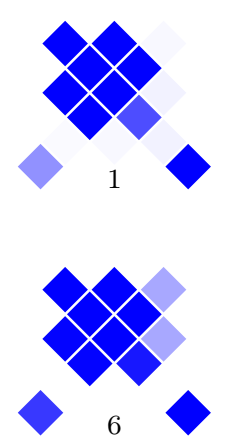

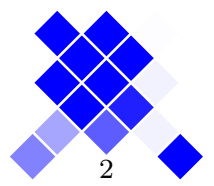

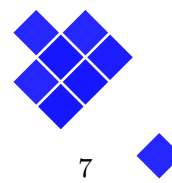

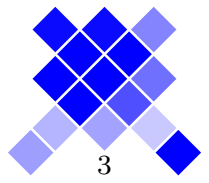

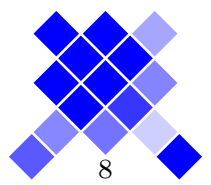

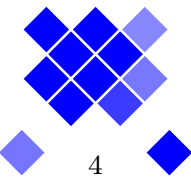

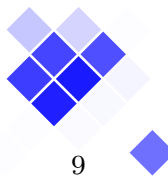

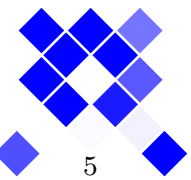

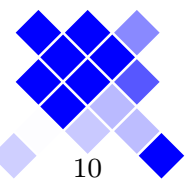

Figure 10: Matcher F-measure signature on the bibliography benchmark (matchers are those presented in $\S 4)$.

For instance, Figure 10 displays the grid for each matcher that will be considered below on the newly generated Benchmark test set. It seems that matcher 7 is only able to provide results if labels are preserved and is not useful otherwise. It is also surprising that matcher 5 works incorrectly when nothing has been altered. In fact, only in this case and only for biblio, this matcher outputs peculiar results which are syntactically incorrect. Finally, it seems that matcher 8 is better at compensating the alteration of both instances and labels than matcher 4 or 6 .

\section{Benchmark assessment}

In order to test the validity of Benchmark principles, we used the test generator to reproduce it with different characteristics. Then, we used 10 different matchers in order to compare the results obtained with the different tests.

Our goal was to explore the properties of Benchmark with respect to what they measure. All our results are given with respect to F-measure. We used F-measure because, given the number of evaluation modalities (random/continuous, four test sets, difficulty, etc.), considering both precision and recall would have complexified the analysis. In particular, the strategy of matchers with respect to trading recall in favour of precision does not help having an independent analysis. We provide in appendix and in the associated material, everything necessary for conducting the same analysis with other measures.

We presented preliminary results in [18] using a limited set of matchers, a reduced set of seed ontologies and a different set of tests. This gave different results from what we obtain now: we still observe strong robustness of results and preservation of difficulty for a matcher across test sets. However, the results allow for identifying a bias in favour of the initial Benchmark test set and the order of matchers across seed ontologies does not seem preserved.

In order to remove the possibility that the obtained results are an artefact of generated tests, we ran the tests five times for each method (continuous and random) and then we computed the average among the obtained results. Likewise, the tests are the same at each run (these tests are 101, 201-202, 221-225, $228,232-233,236-241,246-254,257-262,265-266$, so 94 tests). We decided not to reproduce the ones in which the labels are translated into another language or the ones in which the labels are replaced with their synonyms, because the corresponding alterators are not sufficiently good. The same algorithms with the same parameters have been used for all tests (the tests with the original Benchmark have been run again and restricted to these same tests). 


\subsection{Test sets}

In order to assess the accuracy of the results obtained over the years with the Benchmark test set, we have generated new test sets from different seed ontologies with exactly the same structure and modalities, and compared the results on F-measure. These modalities were chosen because we assume that, since participants have had the opportunity to test their systems with the original Benchmark, their results may be higher on this test set.

New benchmark test sets have been generated from the same seed ontology (bibliography or biblio) and different ontologies of various sizes ${ }^{5}$ :

photography (photo): http://www.co-ode.org/ontolo gies/photography/photography. owl

provenance (prov): http://1sdis.cs.uga.edu/projects /glycomics/propreo/ProPre0-060506.owl

finance : http://www.fadyart.com/ontologies/data/Fi nance.owl

The characteristics of these seed ontologies are summarised in Table 2. Seed ontologies may be selected on the basis of their size, e.g., number of classes, properties, axioms, their domain, e.g., biomedical and tourism ontologies have different vocabularies, or their expressivity. The main criterion for these ones was size.

\begin{tabular}{l|cccc} 
seed & biblio & photo & prov & finance \\
\hline \multicolumn{5}{c}{ Ontology size } \\
\hline classes+prop & 97 & 205 & 431 & 633 \\
instances & 112 & 0 & 46 & 1113 \\
entities & 299 & 205 & 477 & 1746 \\
triples & 1332 & 2236 & 2366 & 21979 \\
\hline Test set generation time (user time on 5 runs) \\
\hline random & 20.79 & 33.46 & 40.05 & 329.52 \\
continuous & 17.37 & 27.89 & 32.26 & 259.13 \\
difficult5 & 21.72 & 33.74 & 39.55 & 336.28 \\
difficult7 & 20.2 & 33.64 & 39.91 & 332.80 \\
difficult9 & 20.3 & 33.23 & 39.37 & 340.17 \\
\hline triple/s & 64 & 67 & 60 & 67
\end{tabular}

Table 2: Progression of the various test sets.

The test sets have been generated using a MacBook Pro Intel Core2Duo 2.66GHz running MacOS X.6.8 (timing performed with the Unix time command).

Table 2 shows that there is a strong constant correlation between the time taken to generate a test set and the number of triples in the test set (and not the number of entities). This is partly due to Jena which forces to go through all triples when renaming entities and when changing the namespace of entities. This performance could be improved, but the linear time is reasonable.

The continuous generation of tests needs around $20 \%$ less time than the random generation. This is a benefit of having a modular generation framework allowing for optimising the generation process (we will see in Section 4.3 that the continuous and random tests provide the same results).

\subsection{Matchers}

The results reported below have been achieved with 10 matchers selected for two reasons:

\footnotetext{
${ }^{5}$ All the ontologies have been retrieved from the web (on 12/2011) and used without any alteration.
} 


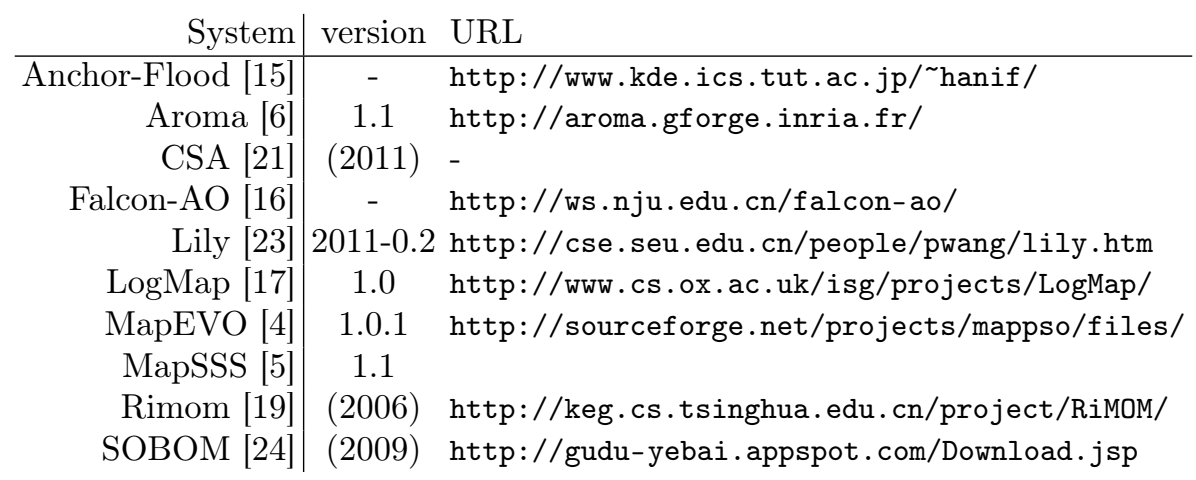

Table 3: Matchers used in the experiments.

- they were available to us, either because they participated to OAEI evaluation under the SEALS platform or because they were generally available;

- they were the fastest systems among a panel of 20 systems, allowing us to run more tests.

Incidentally, these matchers cover 3 out of the top-5 OAEI 2011 performers in terms of F-measure on Benchmark as well as matchers participating in previous OAEI campaigns. These matchers are presented in Table 3. They have been anonymised in the remainder of the paper, because our purpose is to evaluate test generation and not these systems. Systems however, keep the same numbers across all experiments.

In that sense, we will not provide individual timing of matchers. However, the time taken for running these tests evolve with the size of the test set to the extent that they can be differentiated on this basis. For bibliography, the time taken to run the 94 tests in a test set varies from 58 seconds to 48 minutes, while for finance, it varies from 9 minutes to 75 hours with different matchers being the fastest and the slowest (one matcher times out at finance).

\subsection{Robustness}

The first result that can be observed with the test generator is its robustness. Regardless the seed ontology and independently from the generation modality, matchers usually have the same results. All the tests reported here (but those on the original Benchmark) have been obtained over five different generations of each test. However, this is barely useful because the results always reach very close F-measure.

This applies even for non deterministic matchers which may have large variations: these variations do not show up at that level, because these F-measures correspond to the average over 94 ontology matching tasks.

Table 4 shows that the maximum variation that we observe overall on F-measure is .02 on random bibliography. We chose the maximum variation which is much larger than the standard deviation (always less than 1\%). This .02 variation occurs in only one instance for bibliography and one instance for finance. If we consider it on precision or recall, the variation does not exceed .02 on random tests (see Appendix B).

The continuous test sets have more variation: up to .04 in one instance on bibliography and .02 on average. The reason is that, since tests are not independent, any deviation occurring early in the test generation process will be preserved throughout the test set with little opportunity to be compensated.

The variation between random and continuous test sets is so small that it justifies that we concentrate on the random test sets.

In fact, observing large variations (.05) was a sign that something went wrong in the test execution (usually testified by error messages or by the absence of alignments). This is what happened in some of the cancelled matcher results (see Appendix B for details). 


\begin{tabular}{cc|cccccccccc|c} 
& Matcher & 1 & 2 & 3 & 4 & 5 & 6 & 7 & 8 & 9 & 10 & avg \\
\hline biblio & random & .01 & .00 & .00 & .00 & .00 & .02 & .01 & .01 & .00 & .01 & .0 \\
& continuous & .04 & .02 & .01 & .02 & .02 & .01 & .01 & .02 & .02 & .01 & .02 \\
& rand-cont & -.03 & -.02 & -.01 & -.02 & -.02 & +.01 & .0 & -.01 & -.02 & .0 & \\
\hline photo & random & & .00 & & .00 & .00 & & .00 & .01 & .00 & .00 & .0 \\
& continuous & & .01 & & .01 & .01 & & .00 & .01 & .01 & .01 & .01 \\
& rand-cont & & -.01 & & -.01 & -.01 & & .0 & .0 & -.01 & -.01 & \\
\hline \multirow{2}{*}{ prov } & random & .00 & .01 & .01 & .00 & .00 & .00 & .00 & .00 & .00 & .00 & .0 \\
& continuous & .00 & .01 & .01 & .01 & .01 & .00 & .00 & .00 & .00 & .00 & .0 \\
& rand-cont & .0 & .0 & .0 & -.01 & -.01 & .0 & .0 & .0 & .0 & .0 & \\
\hline finance & random & & .01 & .00 & .01 & .00 & .00 & .00 & .00 & .01 & .00 & .0 \\
& continuous & & .00 & .00 & .00 & .00 & .01 & .00 & .01 & .00 & .01 & .0 \\
& rand-cont & & +.01 & .0 & +.01 & .0 & -.01 & .0 & -.01 & +.01 & -.01 &
\end{tabular}

Table 4: Maximum F-measure variation across 5 runs for the different seed ontologies. Empty results correspond to syntax errors explained in Appendix B.

\subsection{Conservativeness}

We noticed that the difficulties encountered by a matcher at a test are preserved across seed ontologies. What we mean is that by looking at the results, test by test, the relative performance at a test for a matcher is preserved across seed ontologies.

So, in order to assess the stability of differences across seed ontologies for a matcher, we have designed a specific indicator. It is relative to a matcher $m$ and a set $S$ of test sets which corresponds to test sets with the same structure generated from different seed ontologies (in our case, $S$ contains biblio, photography, provenance and finance). The indicators are expressed with respect to a particular evaluation measure $M$ : $M\left(m, T_{i}\right)$ is the measure obtained by matcher $m$ on test $T_{i}$. Here we consider F-measure for $M$. These Fmeasures are averaged across values obtained with five independently generated tests for each seed ontology. We still do not use standard deviation because of the difficulty to read it with values between 0 and 1 .

First, we define, for each particular test $T_{i}$, the average of results $A_{m}^{S}(i)$ across all seed ontologies:

$$
A_{m}^{S}(i)=\frac{\sum_{T \in S} M\left(m, T_{i}\right)}{|S|}
$$

This is a level of difficulty of a particular test $\left(T_{i}\right)$ for a particular matcher $(m)$. Then, for each test set, we compute the average difference to this average:

$$
E_{m}^{S}(T)=\frac{\sum_{i=1}^{|T|} M\left(m, T_{i}\right)-A_{m}^{S}(i)}{|T|}
$$

This is a signed difference: it is positive if the test set is easier and negative if it is harder. This is a theoretical measure of the test set $T$ difficulty for a particular matcher $m$ (relative to the others in $S$ ).

We observe on Table 5 that the $E_{m}^{S}$ values seem to induce a general agreement that photography and provenance are harder than bibliography and finance. Indeed, no matcher, but 7, found bibliography more difficult than average, and no matcher but 9 found finance more difficult than average.

Finally, the expected value for $m$ at a particular test $T_{i}$ will be $A_{m}^{S}(i)+E_{m}^{S}(T)$. For measuring the globally observed deviation that a matcher has with these expected measures, we compute:

$$
\Delta_{m}^{S}=\frac{\sum_{T \in S} \sum_{i=1}^{|T|}\left|M\left(m, T_{i}\right)-\left(A_{m}^{S}(i)+E_{m}^{S}(T)\right)\right|}{|S| *|T|}
$$

The result is a cummulative difference because of the absolute value: positive and negative differences are not compensated. A non cummulative difference (without the absolute value) would yield 0 . This is a very strong measure which means that the observed value is on average at $\pm \Delta_{m}^{S} / 2$ of the expected one. 


\begin{tabular}{r|cccccccccc|c} 
Matcher $(m)$ & 1 & 2 & 3 & 4 & 5 & 6 & 7 & 8 & 9 & 10 & avg \\
\hline$E_{m}^{S}($ biblio $)$ & .03 & .10 & .03 & .08 & .11 & .04 & -.07 & .04 & .09 & .01 & .05 \\
$E_{m}^{S}($ photo $)$ & & .00 & & -.05 & -.07 & & .02 & -.05 & .03 & -.08 & -.03 \\
$E_{m}^{S}($ prov $)$ & -.03 & -.15 & -.05 & -.05 & -.07 & -.07 & .02 & -.02 & -.08 & -.01 & -.05 \\
$E_{m}^{S}($ finance $)$ & & .05 & .03 & .04 & .01 & .03 & .02 & .03 & -.04 & .07 & .03 \\
\hline$\Delta_{m}^{S}$ & .03 & .05 & .05 & .06 & .08 & .07 & .02 & .05 & .07 & .05 & .05
\end{tabular}

Table 5: Conservativeness of test difficulty.

\begin{tabular}{|c|c|c|c|c|c|c|c|c|c|c|c|}
\hline 101 & 1 & 2 & J & 4 & 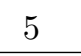 & $\sigma$ & 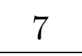 & 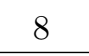 & 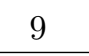 & 10 & avg \\
\hline 0 & .76 & .84 & .85 & .78 & .84 & .76 & .60 & .84 & .40 & .87 & .75 \\
\hline & $c^{2}$ & .81 & .77 & .76 & 84 & .78 & .56 & .82 & .37 & 4 & 72 \\
\hline & .06 & +.03 &.+ & + . & 00 & -.02 & +.04 & 1. & +.03 & 3 & +.0 \\
\hline & .7 & .8 & $=$ & .7 & .84 & .78 & .5 & .82 & .37 & 14 & .72 \\
\hline -1 & +.01 & +.0 & +.4 & +.02 & .00 & -.02 & + . & +.02 & 03 & +.13 & +.0 \\
\hline & & .69 & & .63 & .66 & & .66 & .72 & .25 & .67 & .61 \\
\hline 0 & & +.15 & & +.15 & +.18 & & -.06 & +.12 & +.15 & +.20 & +.13 \\
\hline & 68 & .73 & .67 & .60 & .53 & .67 & .66 & .74 & .12 & 73 & .61 \\
\hline & +.08 & +.11 & +.1 & +.1 & +.3 & +.09 & -.0 & +.10 & +.2 & +.14 & +.14 \\
\hline & & .80 & 7 & .7 & 6 & .77 & .64 & .7 & .1 & .79 & .68 \\
\hline -finance & & +.04 & 06 & +.08 & .17 & -.01 & -.04 & +.05 & +.21 & $+.0 \xi$ & +.07 \\
\hline
\end{tabular}

Table 6: Difference between results obtained through standard benchmarks (reduced to the relevant tests) and newly generated tests.

We can indeed observe the low level of difference between the observed and the expected results $(\Delta)$. All the values are well below .1 variation.

This supports the observation that the results at a test set would provide a signature for a particular matcher, not in the sense that it allows for distinguishing a matcher among others - see the discussion about discriminability in Section $5.2-$, but in the sense that difficulty always evolves in the same way for that matcher.

\subsection{Systematic bias}

Because the original Benchmark has been used for many years with the same ontologies and public reference alignments, it is to be expected that matchers have been particularly trained for this test set.

We compared the results obtained with the original Benchmark to those obtained on newly generated test sets with the same seed ontology, either continuously or randomly, and other seed ontologies. This comparison only covers the 94 tests which are reproduced in the generated test sets (so the results are different than those already published in OAEI results).

Results are synthesised in Table 6. Two of the 10 systems (2 and 3) have many errors in their results which lowers the results they obtain on Benchmark. Only one of the systems (6) has better results on the original Benchmark than on the generated bibliography test sets. Matcher 7, as already observed in $\S 4.4$, is the only system finding the bibliography test set more difficult than the others.

These results correspond to a general bias towards the original Benchmark, since matchers obtain, nearly systematically, better results with it.

There does not seem to be an overfitting to the actual precise tests since the average difference with newly generated test sets from the same seed ontology is only .03, while with other newly generated tests, the advantage is much larger. Hence, matchers seem biased toward the bibliographic ontology rather than towards the initial Benchmark test set itself. 


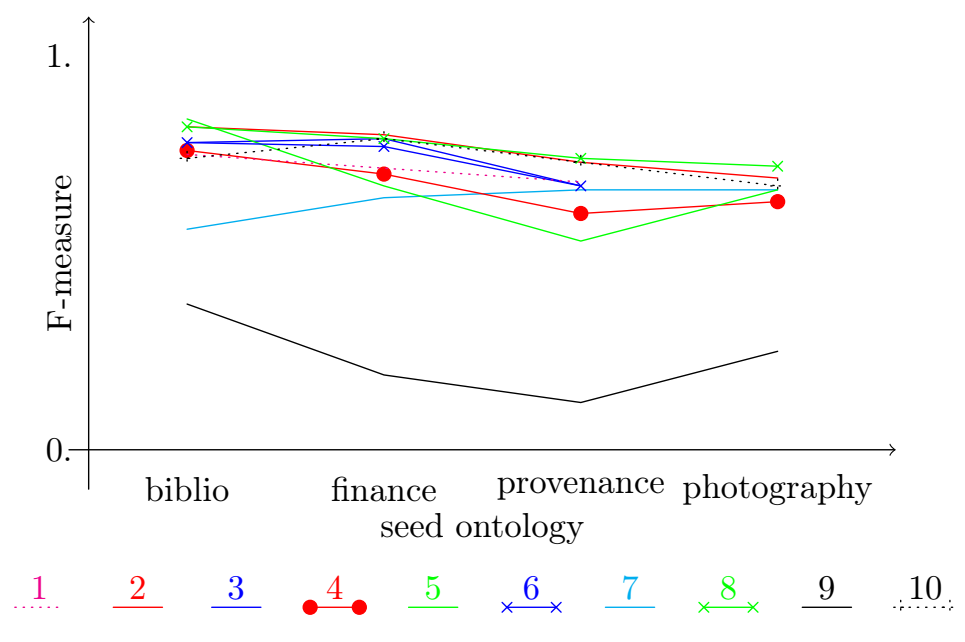

Figure 11: Average F-measure of each system with different seed ontologies. Seed ontologies have not been ordered by size but by average F-measure.

\subsection{Order preservation}

Similarly, we wanted to know if the order between matchers, in terms of F-measure, would be preserved across seed ontologies. Figure 11 shows the evolution of F-measure. Seed ontologies are ordered from the easier to the more difficult (based on average F-measure).

If globally matchers evolve in the same direction, decreasing F-measure with difficulty, the global result obtained with one seed ontology cannot be transposed to another. So, from Figure 11, the order between matchers does not seem preserved. This may be amended when considering the bias for the initial Benchmark test set. Indeed, the three other test sets provide ordered clusters of matchers $(8,2,10$, then 3,6 , 1 , then $7,5,4$ then 9). But it is very difficult to evaluate precisely this order preservation, because matcher results are often very close. This is related to the lack of discriminability between matchers. Figure 11 shows that for these tests, all the matchers evolve very closely (except for the black outlier). Hence, if one wants to draw conclusions for comparing matchers, it is necessary to use more discriminant tests.

\subsection{Conclusion}

This study revealed several properties of the Benchmark setting for evaluating ontology matchers:

Robustness The results will be the same for a matcher across different randomly generated test sets from the same ontology. This is also true when the test set is generated in a random or continuous way. This provides confidence in the generator and in the initial Benchmark test set.

Conservativeness Benchmark is good for studying matcher behaviour, and even relative matcher behaviour, because matcher profiles with respect to test difficulty is preserved. Hence, independently from the seed ontology, the difficult cases for a matcher will remain the same tests.

Bias It is better to compare systems on the basis of seed ontologies different from the original one. Arguably, matchers are biased towards this particular test set or domain albeit to different extents.

Order The order between matchers is not preserved across seed ontologies. Hence, Benchmark is not good for identifying the best matcher. However, having the ability to generate a test set from an ontology allows for selecting the one suited for a particular test set.

This shows that Benchmark is indeed adequate for finding the profile of a matcher, but not for comparing matchers. Matcher comparison will depend on the particular situation, e.g., the seed ontology.

In addition, it is difficult to have a definitive judgement about the preservation of matcher order across different seed ontologies, because the tests do not allow for sufficient discrimination. Hence, we consider this problem in the remainder. 


\section{Difficulty and discriminability}

One of the drawbacks of the initial Benchmark test set is its lack of difficulty and discriminability (§1). A new test generator allows for addressing such drawbacks in an interesting way. As mentioned, Benchmark, being focussed on paving the problem space regularly, contains many easy tests. We present here two ways to use the test generator in order to generate more difficult (§5.1) and discriminant (§5.2) test sets.

\subsection{Increasing difficulty}

The first way to increase difficulty has consisted of altering differently the labels in the test sets. Instead of having a regular alteration of 20,40,60, 80 and $100 \%$ of the labels, we decided to modify the benchmark generator so that it alters more radically the labels depending on the required test difficulty. Hence, the alteration $p_{\alpha}(i)$ at level $i$ for difficulty $\alpha$ is:

$$
p_{\alpha}(i)=1-(1-\alpha)^{i}
$$

The corresponding values are given in Table 7 .

\begin{tabular}{c|cccc}
$i \backslash \alpha$ & 0. & .5 & .7 & .9 \\
\hline 0 & $0 \%$ & $0 \%$ & $0 \%$ & $0 \%$ \\
1 & $20 \%$ & $50 \%$ & $70 \%$ & $90 \%$ \\
2 & $40 \%$ & $75 \%$ & $91 \%$ & $100 \%$ \\
3 & $60 \%$ & $87 \%$ & $97 \%$ & $100 \%$ \\
4 & $80 \%$ & $94 \%$ & $99 \%$ & $100 \%$ \\
5 & $100 \%$ & $97 \%$ & $100 \%$ & $100 \%$
\end{tabular}

Table 7: Percentage of altered labels depending on each of the modalities (the first modality does not follow the $p_{\alpha}(i)$ formula).

This kind of modification in the test generation process could be applied to any type of alteration (see $\S 5.2$ ), but this way preserves the structure of the original Benchmark, so we used it for comparison purposes.

\subsubsection{Setting}

We have generated three new test sets, based on the same ontologies, with three new difficulty levels $(.5$, .7 and .9) using the random generation modality. This has been possible by simply introducing a parameter in the BenchmarkGenerator class which generates the Benchmark test set (the implementation of Figure 5 and Figure 6).

The same matchers as above $(\S 4.2)$ have been used on these tests.

\subsubsection{Results}

Figure 12 shows that the proposed technique indeed results in more difficult tests. All systems have decreasing F-measure as difficulty increases. This is correctly observed across all seed ontologies.

This shows that the difficulty of tests can be systematically and uniformly controlled by the test generation process. However, this does not increase discrimination: matchers remain as close as in the initial test set. Other techniques are necessary for increasing discriminability.

\subsection{Increasing discriminability}

Looking at results on bibliography (iteration 0 of Figure 14), it seems clear that the tests discriminate between matchers $5,1,10,7$, and 9 . These are also clearly distinguishable from the group of matchers 6,8 , 4, 3 and 2. But among this latter group, the marchers are very close to each others. In particular, 8 and 4 are barely distinguishable. We describe a general use of the generator for increasing discriminability of a given test set. 

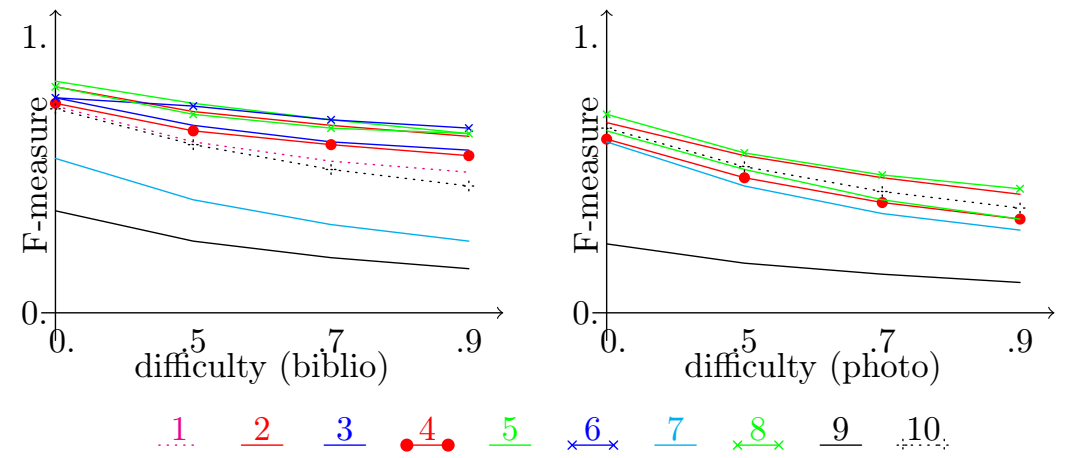

Figure 12: Average F-measure of each system with increasing difficulty $(0 ., .5, .7, .9)$ tests on the bibliography (left) and photography (right) seed ontologies.

If we know what is discrimination between two matchers (their F-measure is at a large distance), we do not know what it is for several matchers at once. Hence, if discrimination seems a desirable property of a test set, the notion of discrimination put forward in [13] would gain in being formally defined so that we could measure that test sets indeed discriminate.

We thus designed a measure based on the spread of values which we call ideal spread distance. It is represented by the difference between the actual spread of values and a uniform spread, so for results of a set $S$ of matchers:

$$
i s d(i)=\sum_{k=0}^{|S|-1}\left|M\left(\iota(k), T_{i}\right)-k /(|S|-1)\right|
$$

with $\iota$ a function ordering the matchers by increasing F-measure. This value goes from $|S| / 2(5$ in our case) when all measures are the same to 0 . when they are equi-distributed over the unit interval. The ideal spread distance is used here as a possible discriminability indicator, other definitions may be retained.

One may argue that it is possible to increase discrimination by just spreading evenly the result values to the unit interval, preserving the order. This would indeed, reduce the ideal spread distance to 0 ., but then (a) discrimination would be useless since it would only amplify a measure that was already available (no information gain), and (b) the obtained values would be meaningless (these would be ordinals).

This is why we try to proceed by adding new tests which are chosen on the basis of discrimination expectation and not an artificial guarantee of discrimination. The difficulty is to find such tests.

The process is the following:

1. Generate the full alteration space on all $(n)$ dimensions with the simplest parameters: $0 \%$ and $100 \%$ alteration. This is $N_{0}=2^{n}$ tests.

2. Run a set of matchers and compute a value, e.g., average F-measure, for each tests which has no such value.

3. Select the adjacent pair of tests with the highest difference between these values, if a threshold is reached, then exit.

4. Generate an intermediate test with the following parameters: if the two tests are characterised by parameter vectors $\left\langle p_{1}, \ldots p_{n}\right\rangle$ and $\left\langle p_{1}^{\prime}, \ldots p_{n}^{\prime}\right\rangle$, then generate the test with parameters $\left\langle\frac{p_{1}+p_{1}^{\prime}}{2}, \ldots \frac{p_{n}+p_{n}^{\prime}}{2}\right\rangle$.

5. Optionally, complete (close) the set of tests so that for any pair of tests $\left\langle p_{1}, \ldots p_{n}\right\rangle$ and $\left\langle p_{1}^{\prime}, \ldots p_{n}^{\prime}\right\rangle$, there exists $\left\langle\min \left(p_{1}, p_{1}^{\prime}\right), \ldots \min \left(p_{n}, p_{n}^{\prime}\right)\right\rangle$ and $\left\langle\max \left(p_{1}, p_{1}^{\prime}\right), \ldots \max \left(p_{n}, p_{n}^{\prime}\right)\right\rangle$ within the test set.

6. Go to Step 2.

Figure 13 provides an example of running such a process.

This process can itself be parameterised in several manners in addition to the seed ontology and the set of matchers: 


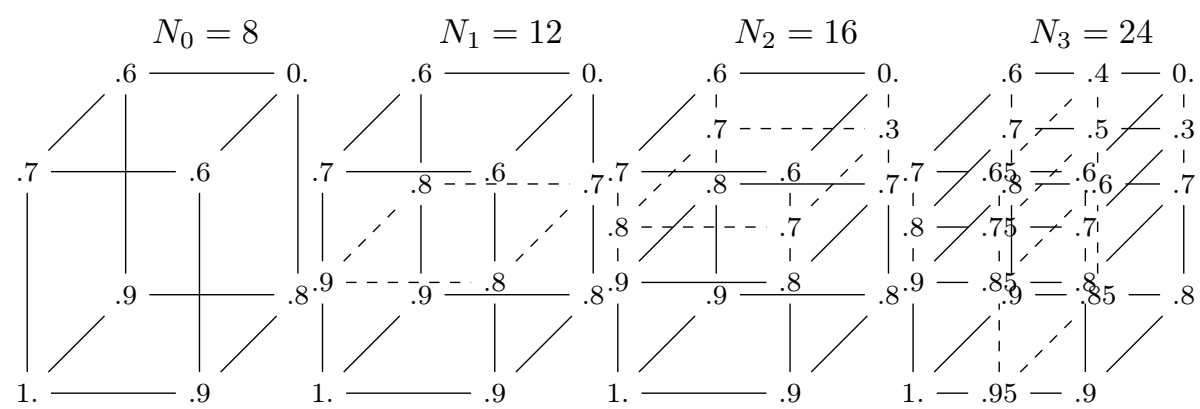

Figure 13: Iterative execution of the adaptive test generation (3 iterations). Each vertex is labelled by average F-measure from which difference at each edge is computed.

- The number of dimensions;

- The type of values computed: this may be average F-measure or other measures, such as standard deviation or the difference between the highest and the lowest F-measure in the test;

- The output threshold may be a number of iterations, a maximum or minimum value for the previous measure or a global evaluation of difficulty or discriminability on the test set;

- The completion or not of the test set;

- The continuous or random generation of the new tests.

\subsubsection{Setting}

Such an adaptive process can be implemented with the given test generation architecture. The difficulty of this particular generator is that generation depends on test results. Hence it is necessary to combine generation, matching and evaluation. Although the Alignment API allows for such a combination, we implemented the computation by hand, i.e., we generated a priori all the necessary tests and used the relevant ones at each iteration.

We generated test sets of 125 tests corresponding to 5 levels of alteration on three dimension $\left(5^{3}=125\right)$, already present in the initial Benchmark tests:

1. property removal;

2. comment removal;

3. label scrambling.

Five such test sets based on the bibliography seed ontology were generated. The initial iteration (0) of Figure 14 corresponds to the use of 8 tests offering either $0 \%$ or $100 \%$ alteration on each dimension. Increasing discrimination was attempted by adding new tests.

We ran the same 10 matchers as above $(\S 4.2)$ on these new test sets and simulated the effect of the above process.

\subsubsection{Results}

The computations necessary for deciding which dimension to further develop were initially based on two different criteria:

- largest difference in the difference between the maximum and the minimum F-measure (across all systems);

- largest difference in standard deviation (between F-measures).

We compared the results obtained by selecting the dimension based on the largest value between two vertices or the largest value on average between all vertices in which the alteration matters. This provided the same result. 

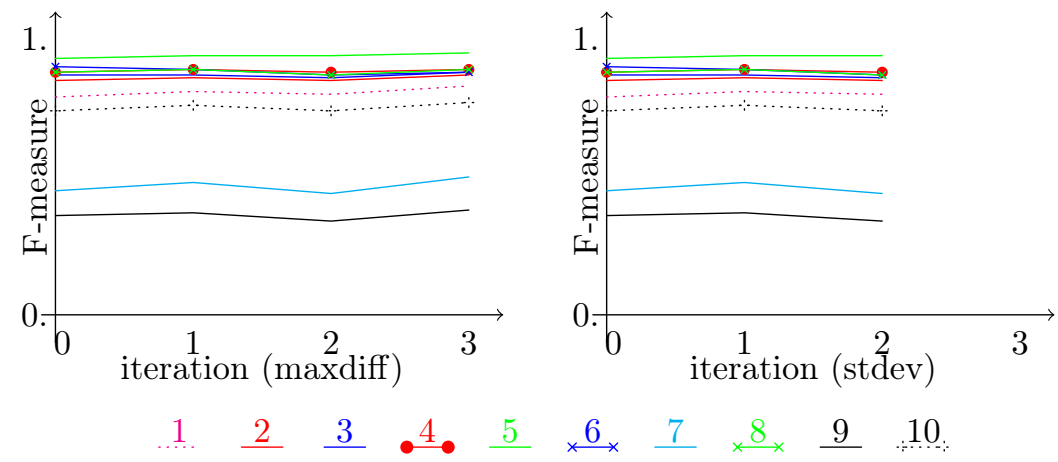

Figure 14: Average F-measure at different iterations of the discriminability procedures with the bibliography seed ontology when difficult tests are added on a whole dimension at once (left: with maximum difference, right: with standard deviation). Standard deviation stops after two iterations because it always selects the same dimension to further develop.
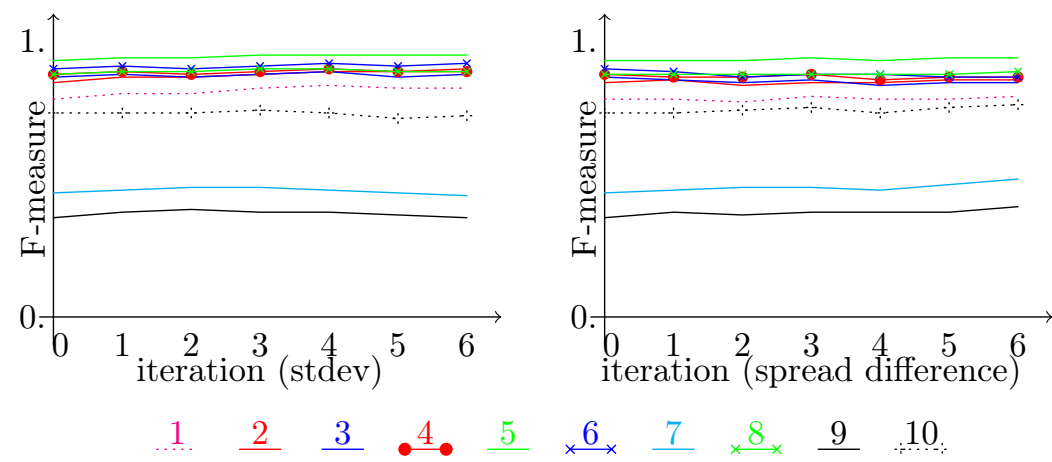

Figure 15: Average F-measure at different iterations of the discriminability procedures with the bibliography seed ontology when difficult tests are added one by one (left: with standard deviation; right: with the spread difference measure).

In each case, we iterated three times. In most cases, the selected dimension was the label scrambling dimension.

The results, provided in Figure 14, show that this approach does not allow us to discriminate much more the matchers than the initial tests. The ideal spread distance of the initial state (iteration 0 ) is 2.78 , the resulting values for maxdiff and stdev are respectively 2.94 and 2.72 . Hence the proposed procedure does not succeed in significantly increasing discrimination.

We tried to directly use the ideal spread distance in order to identify the most promising dimension to develop. Indeed, it is possible to compute the ideal spread distance for any single test and when the difference between this measure is high for two adjacent tests, an intermediate test may raise the overall spread. When applying the ideal spread distance to the tests with either $0 \%$ or $100 \%$ alteration, the obtained value is maximal (4.83) when nothing has been altered and minimal (1.65) when everything has been altered. However, there are lower values in the other generated tests.

Using the ideal spread distance with the closing modality provides comparable results to those of Figure 14 for the maximum difference criterion (left). Figure 15 displays the results of the procedure when tests are added one by one, instead of dimension per dimension (closing modality) for standard deviation and ideal spread distance difference. In both cases, the final ideal spread distance was 2.88 , failing again to improve on this measure. 


\begin{tabular}{c|ccc|ccc} 
iteration & 4 & 8 & st.dev. & 4 & 8 & st.dev. \\
\hline 0 & .8825 & .8825 & .0000 & .8825 & .8825 & .0000 \\
1 & .8893 & .8892 & .0001 & .8720 & .8842 & .0086 \\
2 & .9040 & .9098 & .0041 & .8754 & .8926 & .0122 \\
3 & & & & .8684 & .8900 & .0153 \\
4 & & & & .8412 & .8657 & .0173 \\
5 & & & & .8534 & .8718 & .0131 \\
6 & & & & .8639 & .8796 & .0111
\end{tabular}

Table 8: Increased discrimination between two systems based on standard deviation by adding the single most discriminating dimension (left) or the most discriminating test (right) at each iteration.

We performed the same tests in order to better discriminate between only two matchers. In this case, using the maximum difference or the standard deviation of average F-measure gave the same results. We chose to discriminate between the two matchers with the closest average F-measure, i.e., where discrimination is needed. We obtained a better discrimination as is shown in Table 8 by the increase of average standard deviation. However, this increase in discrimination brought a difference in which system is best. When working specifically with these two systems, adding blindly the test that increases standard deviation makes that 4 has a higher F-measure than 8 at iteration 1, then 8 has a higher F-measure than 4 at iteration 2 . In fact, this could change at further iterations. Similar observations were possible with the other discrimination experiments.

Indeed, modifying tests for the sole purpose of increasing discriminability does not guarantee that results are preserved. This is a problem with putting the requirement of discrimination above other requirements such as the ability to measure something. As we have already mentioned, some systems are so close to each other, not only in terms of performance but in terms of design, that discriminating between them is not informative.

\section{Conclusion}

In this paper we have brought three contributions to ontology matching evaluation:

- an ontology alignment test generator which is extensible and flexible;

- extensive experiments with the Benchmark modality used in OAEI, showing that it indeed measures permanent properties of matchers;

- Benchmark-like tests with improved variability and difficulty.

We have developed a test generator which follows a simple modular architecture and API. This generator does not depend on the seed ontology. It allows for different modifications at each run of the program and the set of input parameters can be adjusted in order to cover the problem space with any precision. It is also extensible and flexible: it can be extended by adding new ontology alterators and it can be used for generating individual tests with controlled characteristics as well as full test sets.

This generator has been used for generating test sets on the model of Benchmark with different seed ontologies and different modalities. This largely improved the variability of generated tests. Some of these generated tests have been used in OAEI 2011. From these new generated test sets, we have been able to assess (a) the robustness of evaluation results which remains the same for differently generated test sets and (b) the preservation of the relative difficulty across test sets generated with different seed ontologies. On the other hand, we also identified (c) an apparent bias towards the initial Benchmark test set and seed ontology, and $(\mathrm{d})$ a poor preservation of the order between matchers across seed ontologies.

These observations confirm that the Benchmark test set is suited for finding the strengths and weaknesses of matchers, but not suited for ranking matchers universally. This remark may also be related to the relative 
proximity of results provided by matchers at such evaluations. It may be due to the intrinsic proximity of matchers or to the lack of discriminability of the proposed test sets.

We have used the same test generator to address the problems of difficulty and discriminability by generating specifically designed test sets. This has shown that increasing test difficulty is not a problem, but increasing discriminability proved to be more difficult than expected. Indeed, improving discriminability through the addition of new tests in a test set would require a precise definition of what is expected, which would lead to a practical evaluation measure. All those that we tried (standard deviation, maximum span, average distance) did not provide convincing results.

Moreover, discrimination, taken as an absolute property may lead to twist results so that they discriminate more instead of providing an objective assessment of matcher quality. Hence, we think that discriminability should not be taken as an absolute criterion for test sets. It is more important to have test sets providing meaningful and interpretable results than discriminant ones.

In conclusion, a test generator as the one presented in this paper is a valuable tool for introducing variability in benchmarks. In addition, assessing the properties of generated tests is important to understand what is evaluated and exposing benchmarks to critical discussion. This can be achieved theoretically or experimentally. In the latter case, a flexible test generator is precious.

This test generation framework has been used for generating new test cases OAEI 2011.5 and OAEI 20012 campaigns, focusing in particular on scalability. We also consider extending it for generating networks of ontologies instead of pairs of ontologies, and further analysing the notion of test hardness applied to the ontology matching problem.

\section{Acknowledgements}

This work has been partially supported by the SEALS project (IST-2009-238975).

\section{Appendix A. Additional material}

\section{Appendix A.1. Directory structure}

In order to allow for reproducibility and result analysis, we provide the following resources:

ontologies/ The seed ontologies used for generating these tests are those of $§ 4.1$.

origin/ The original Benchmark test set.

dataset/seed/modality/run/test The different generated test sets (onto.rdf and refalign.rdf) and results provided by matchers: seed is the ontology name, modality is one of benchmark, continuous, disc, difficult 5,7 , or 9 , run is the number of the generated test set ( 0 is the initial bibliography test), and test the particular test number.

results/ The results computed and aggregated (in LaTeX, html and as spreadsheets).

Various scripts for regenerating or manipulating these data are also available.

These test sets and results are available at ftp://ftp.inrialpes.fr/pub/exmo/datasets/bench2012. zip (the zip file is around $700 \mathrm{MB}$ ).

\section{Appendix A.2. Test generation}

The test generator for generating new independent test sets is available in the Alignment API 4.3 (http: //alignapi.gforge.inria.fr).

Tests may be generated from command lines with the following script:

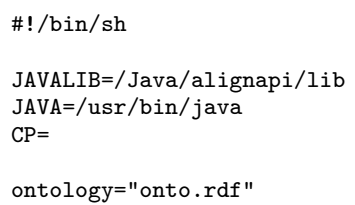




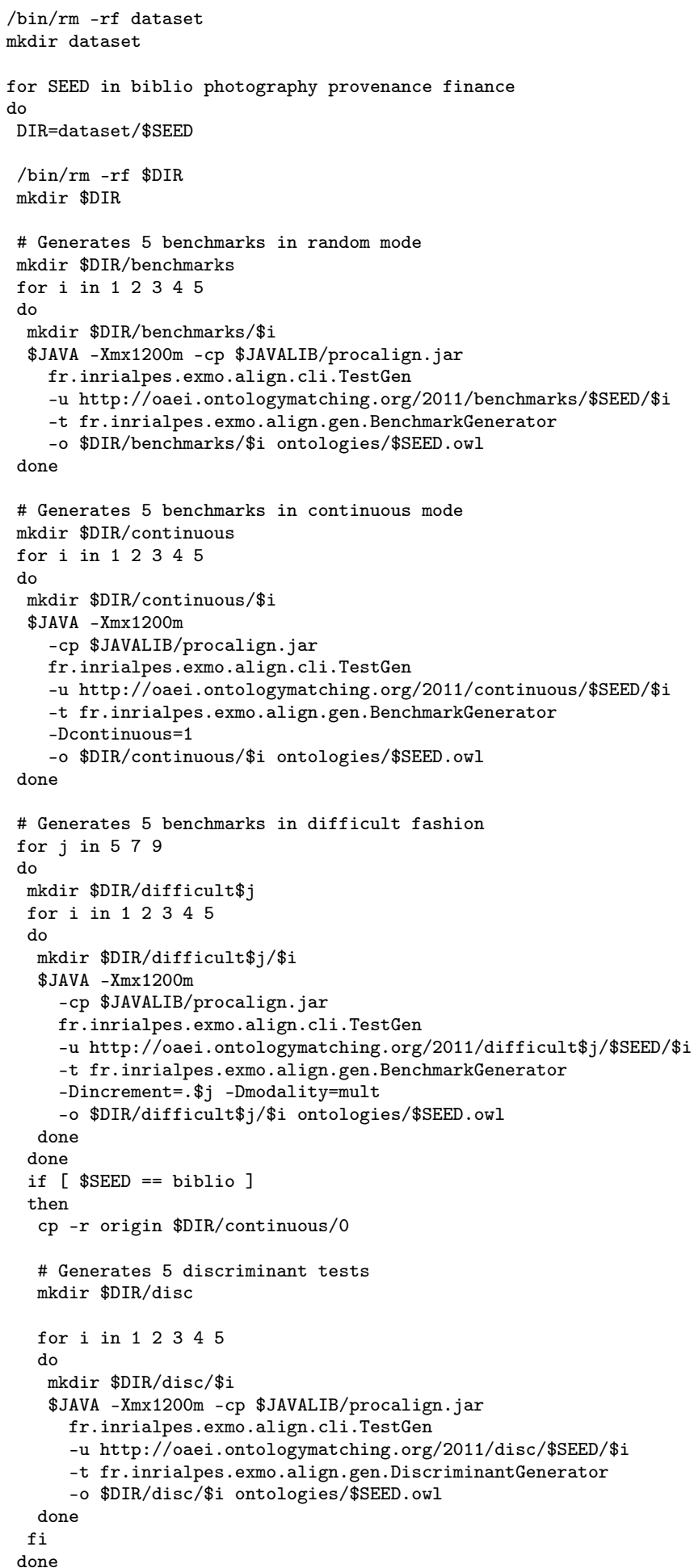

We do not provide the matchers because we do not necessarily have the right to do so; we indicated in Table 3 where they could be found.

When a matcher is available under the Alignment API, instructions for running the matcher are: 
\$JAVA - cp \$JAVALIB/procalign.jar

fr.inrialpes.exmo.align.util.GroupAlign

-o edna -n file://\$TESTDIR/101/onto.rdf

-i fr.inrialpes.exmo.align.impl.method.StringDistAlignment

-DstringFunction=levenshteinDistance -Dnoinst=1

Not all matchers are available under this API. We ran the evaluations through the SEALS platform client ${ }^{6}$.

\section{Appendix A.4. Evaluation}

We also provide the evaluation results (precision, recall, F-measure) computed from these results in the results directory. These computation can be achieved through the Alignment API by:

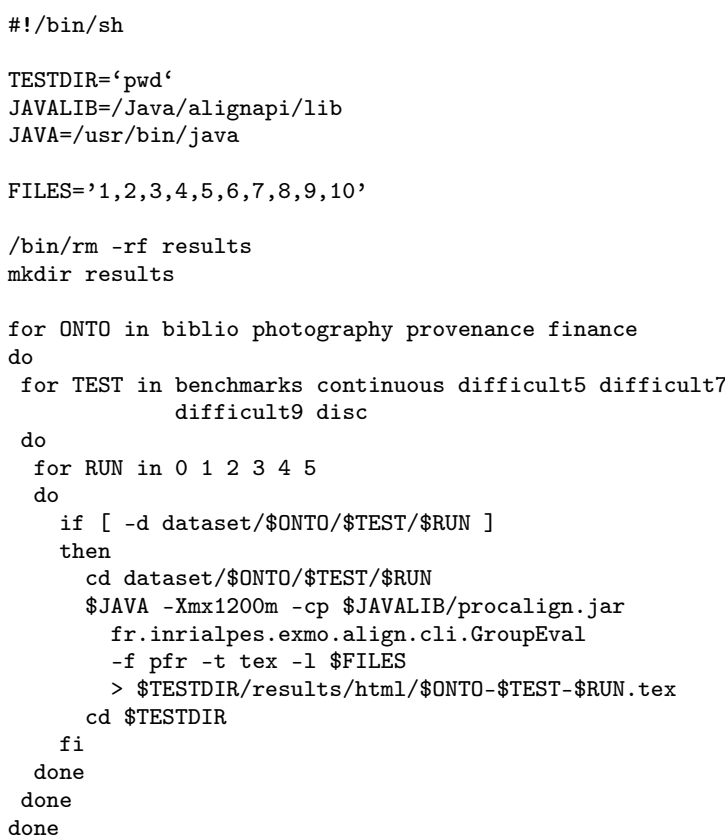

Appendix A.5. Statistics

\begin{tabular}{|c|c|c|c|c|c|c|c|}
\hline & 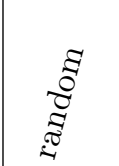 & 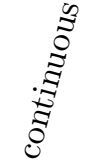 & $\underbrace{20}_{i}$ & $\stackrel{\wedge}{\sharp}$ & : & $\ddot{\pi}$ & $\underset{\mathbb{I}}{\mathbb{D}}$ \\
\hline biblio & $5 \times 10$ & $6 \times 10$ & $5 \times 10$ & $5 \times 10$ & $5 \times 10$ & $5 \times 10$ & $\overline{310}$ \\
\hline lotography & $5 \times 7$ & $5 \times 7$ & $5 \times 7$ & $5 \times 7$ & $5 \times 7$ & & 175 \\
\hline provenance & $5 \times 10$ & $5 \times 10$ & $2 \times 10$ & $2 \times 10$ & $2 \times 10$ & & 16 \\
\hline finance & $5 \times 9$ & $2 \times 9$ & $2 \times 9$ & $2 \times 9$ & $2 \times 9$ & & 117 \\
\hline total & 180 & 163 & 123 & 123 & 123 & 50 & 02 \\
\hline
\end{tabular}

Table A.9: Number of tests on which this study is based: $712 \times 94+50 \times 125=73178$ matching tests.

Table A.9 summarises the different result sets on which this paper is based. This represents 712 test sets containing 94 elementary matching tasks, plus 5 discriminant test sets of 125 matching tasks, hence 73178 elementary matching tasks. In reality, we run far more tests than this number: cancelled results from the following table have been computed (and are provided in the zip file) and many more tests have been performed in the preparatory phase.

${ }^{6}$ http://oaei.ontologymatching.org/2011.5/seals-eval.html 


\section{Appendix B. Aggregated precision/F-measure/ recall per test suite}

Table B.10, B.11, B.12 and B.13 provide aggregated precision, F-measure and recall for all generated test sets. We tried to ensure that we had as much results as possible but the tests taking so long in some instances, it was not possible to run them as many times as we had wished for. This is the reason why the largest tests were not run 5 times.

There have been difficulties with several systems, the effect of which has been cancelled. In particular, we had problems with:

- One system (1) failing completely to run on finance due to time out;

- Two systems (1 and 6) failing to run on photography due to incorrect URIs in the ontologies (ignored by other systems);

- One system (3) delivering in some cases only, significantly different results on photography due to the same incorrect URIs (definitely a bug);

- Two systems (2 and 3) failing to run on bibliography from test 224 due to a Jena error: "Property not found" exception: rdf:rest.

In the first three cases, we decided to ignore the results (they are provided in the zip). They were due to our methodology to not alter ontologies and the late discovery of incorrect URIs (with fragments like $f / 22$ or $24 \times 36$ ) which were silently but incorrectly parsed by Jena.

\section{Appendix C. Description of elementary alterators}

We provide a description of the elementary alterators currently implemented in the Alignment API. Further details about how to use them are available at http://align api.gforge.inria.fr/testgen. html. A more elaborate description of how they were used in Benchmark is available at http://oaei. ontologymatching.org/tests/.

The functional description is given at the level of OWL, however, the actual implementation largely differs by taking advantage of different internal structures referring to an actual ontology model. Hence, sometimes modifications are applied on the OWL structure, and sometimes they are applied at the level of triples. The final model and alignments are generated at the end of the process. This is only at the end of the process that the entities are assigned a final URI.

Parameters are key-value pairs defining the degree or modalities of alteration. The priority is expressed by a level such that modifications of lower level must be applied before modifications of higher level. All classes are in the fr.inrialpes.exmo.align.gen.alt package.

\section{EmptyModification}

Class: EmptyModification

No parameters

Result: generates a test in which the initial ontology is not altered. This test is used in test sets, for generating an initial identity alignment.

Functional description: For each named class and each named property in the input ontology, creates a correspondence with equivalence between this entity and itself.

Priority: 0

\section{RemoveClasses}

Class: RemoveClasses

Parameters: removeClasses $=p$

Result: removes $p \%$ of classes.

Functional description: Selects randomly $p \%$ of classes to suppress from the ontology. For each of these classes, attaches, directly, its subclasses to its superclasses (including constraints, i.e., anonymous Restriction classes); attaches, directly, its instances to its superclasses; converts all restrictions involving it 
(allValuesFrom, someValueFrom) with its superclasses; converts all property restrictions involving it (domain, range) with its superclasses; removes the class. Updates the alignment by suppressing all correspondences involving the suppressed classes.

Priority: 1

\section{RemoveComments}

Class: RemoveComments

Parameters: removeComments $=p$

Result: removes $p \%$ of comments.

Functional description: Selects, randomly and independently from the ontology, $p \%$ of classes, properties and individuals whose comments will be suppressed. Removes the comments of each selected entity. The alignment is not modified.

Priority: 2

\section{RemoveIndividuals}

Class: RemoveIndividuals

Parameters: removeIndividuals $=p$

Result: removes $p \%$ of individuals.

Functional description: Selects randomly $p \%$ of individuals to suppress from the ontology. Suppresses from the ontology model all statements using these individuals, either as subject or object. The alignment is not modified, because it does not contain individuals.

Priority: 2

\section{RemoveProperties}

Class: RemoveProperties

Parameters: removeProperties $=p$

Result: removes $p \%$ of properties.

Functional description: Selects randomly $p \%$ of properties to suppress from the ontology. For each of these properties, removes all restrictions (allValuesFrom, someValues From) involving it, removes all assertions involving it. Updates the alignment by suppressing all correspondences involving the suppressed properties. Priority: 2

\section{RemoveRestrictions}

Class: RemoveRestrictions

Parameters: removeRestrictions $=p$

Result: removes $p \%$ of restriction.

Functional description: Selects randomly $p \%$ of property restrictions to suppress from the ontology. For each of these restrictions, removes it from the model. The alignment is not modified.

Priority: 3

RenameClasses

Class: RenameClasses

Parameters: renameClasses $=p$, renameMethod $=m$

Result: renames $p \%$ of classes depending on the requested method $m$ (random strings, translation, synonyms) [default: random]

Functional description: Selects randomly $p \%$ of classes from the ontology to rename. For each of these classes, creates a new name depending on the selected $m$ and replace its rdfs:label by the new name. For each triple of the model, all URI fragments are replaced if necessary by the corresponding new name. Each correspondence of the alignment involving a renamed class is replaced by the corresponding new URI.

Priority: 4 


\section{RenameProperties}

Class: RenameProperties

Parameters: renameProperties $=p$, renameMethod $=m$

Result: renames $p \%$ of properties depending on the requested method $m$ (random strings, translation, synonyms) [default: random]

Functional description: Selects randomly $p \%$ of properties from the ontology to rename. For each of these properties, creates a new name depending on the selected $m$. For each triple of the model, all URI fragments are replaced if necessary by the corresponding new name. Each correspondence of the alignment involving a renamed property is replaced by the corresponding new URI.

Priority: 4

AddClasses

Class: AddClasses

Parameters: addClasses $=p$

Result: adds $p \%$ classes.

Functional description: Selects randomly $p \%$ of classes from the ontology to add a subclass to. For each of these classes, generates randomly a new name; creates a new subclass of the selected class with this name. The alignment is not modified.

Priority: 5

\section{AddProperties}

Class: AddProperties

Parameters: addProperties $=p$

Result: adds $p \%$ properties.

Functional description: Selects randomly $p \%$ of properties from the ontology to add. For each of these properties, generates randomly a new name. For half of these new properties, creates an ObjectProperty: selects randomly a domain and range among the classes and assign them to this property. For the other half of these new properties, creates a DatatypeProperty: selects randomly a domain among the classes and assign it to this property. range is set to xsd:string. The alignment is not modified.

Priority: 5

\section{FlattenLevel}

Class: FlattenLevel

Parameters: levelFlattened $=n$

Result: removes all classes of level $n$ (their subclasses are directly connected to their superclasses).

Functional description: For each class of that level, attaches, directly, its subclasses to its superclasses (including constraints, i.e., anonymous Restriction classes); attaches, directly, its instances to its superclasses; converts all restrictions involving it (allValuesFrom, someValueFrom) with its superclasses; converts all property restrictions involving it (domain, range) with its superclasses; removes the class. Updates the alignment by suppressing all correspondences involving the suppressed classes.

Priority: 6

SuppressHierarchy

Class: SuppressHierarchy

No parameter

Result: suppresses all subclasses relations (but to Thing)

Functional description: Suppresses all subClass0f assertions towards other named classes (assertions towards Restrictions remain). The alignment is not modified.

Priority: 7 


\section{References}

[1] Bogdan Alexe, Wang-Chiew Tan, and Yannis Velegrakis. STBenchmark: towards a benchmark for mapping systems. In Proc. 34th Very Large Databases conference (VLDB), Auckland (NZ), pages 230-244, 2008.

[2] Zohra Bellahsene, Angela Bonifati, Fabien Duchateau, and Yannis Velegrakis. On evaluating schema matching and mapping. In Zohra Bellahsene, Angela Bonifati, and Erhard Rahm, editors, Schema matching and mapping, chapter 9, pages 253-291. Springer-Verlag, Heidelberg (DE), 2011.

[3] Paolo Besana. Predicting the content of peer-to-peer interactions. PhD thesis, University of Edinburgh, 2009.

[4] Jurgen Bock, Carsten Danschel, and Matthias Stumpp. MapPSO and MapEVO results for OAEI 2011. In Proc. 6th ISWC workshop on ontology matching (OM), Bonn (DE), pages 179-183, 2011.

[5] Michelle Cheatham. MapSSS results for OAEI 2011. In Proc. 6th ISWC workshop on ontology matching (OM), Bonn (DE), pages 184-189, 2011.

[6] Jérôme David, Fabrice Guillet, and Henri Briand. Association rule ontology matching approach. International Journal of Semantic Web and Information Systems, 3(2):27-49, 2007.

[7] Fabien Duchateau, Zohra Bellahsene, and Ela Hunt. XBenchMatch: a benchmark for XML schema matching tools. In Proc. 33rd Very Large Databases (VLDB) demo track, Wien (AT), pages 1318-1321, 2007.

[8] Jérôme Euzenat, Marc Ehrig, Anja Jentzsch, Malgorzata Mochol, and Pavel Shvaiko. Case-based recommendation of matching tools and techniques. deliverable 1.2.2.2.1, Knowledge web, 2006.

[9] Jérôme Euzenat, Christian Meilicke, Heiner Stuckenschmidt, Pavel Shvaiko, and Cássia Trojahn dos Santos. Ontology alignment evaluation initiative: Six years of experience. Journal on Data Semantics, XV:158-192, 2011.

[10] Jérôme Euzenat and Pavel Shvaiko. Ontology matching. Springer-Verlag, Heidelberg (DE), 2007.

[11] Alfio Ferrara, Davide Lorusso, Stefano Montanelli, and Gaia Varese. Towards a benchmark for instance matching. In Proc. 3rd ISWC Ontology matching workshop, Karlsruhe (DE), pages 37-48, 2008.

[12] Alfio Ferrara, Stefano Montanelli, Jan Noessner, and Heiner Stuckenschmidt. Benchmarking matching applications on the semantic web. In Proc. 8th Extended Semantic Web Conference (ESWC), Herssounisos (GR), number 6644 in Lecture notes in computer science, pages 108-122, 2011.

[13] Fausto Giunchiglia, Mikalai Yatskevich, Paolo Avesani, and Pavel Shvaiko. A large scale dataset for the evaluation of ontology matching systems. Knowledge engineering review, 24(2):137-157, 2009.

[14] Yuanbo Guo, Abir Qasem, Zhengxiang Pan, and Jeff Heflin. A requirements driven framework for benchmarking semantic web knowledge base systems. IEEE Transactions on Knowledge and Data Engineering, 19(2):297-309, 2007.

[15] Md. Seddiqui Hanif and Masaki Aono. An efficient and scalable algorithm for segmented alignment of ontologies of arbitrary size. Journal of web semantics, 7(4):344-356, 2009.

[16] Wei Hu and Yuzhong Qu. Falcon-AO: A practical ontology matching system. Journal of web semantics, 6:237-239, September 2008.

[17] Ernesto Jiménez-Ruiz and Bernardo Cuenca Grau. LogMap: Logic-based and scalable ontology matching. In Proc. 10th International Semantic Web Conference (ISWC), Bonn (DE), pages 273-288, 2011.

[18] Maria Roşoiu, Cássia Trojahn dos Santos, and Jérôme Euzenat. Ontology matching benchmarks: generation and evaluation. In Proc. 6th ISWC workshop on ontology matching (OM), Bonn (DE), pages 73-84, 2011.

[19] Jie Tang, Juanzi Li, Bangyong Liang, Xiaotong Huang, Yi Li, and Kehong Wang. Using bayesian decision for ontology mapping. Journal of web semantics, 4(4):243-262, 2006.

[20] Rémi Tournaire. Découverte automatique de correspondances entre ontologies. PhD thesis, Université de Grenoble, 2010.

[21] Quang-Vinh Tran, Ryutaro Ichise, and Bao-Quoc Ho. Cluster-based similarity aggregation for ontology matching. In Proc. 6th ISWC workshop on ontology matching (OM), Bonn (DE), pages 142-147, October 2011.

[22] Lorenzino Vaccari, Pavel Shvaiko, Juan Pane, Paolo Besana, and Maurizio Marchese. An evaluation of ontology matching in geo-service applications. Geoinformatica, 16(1):31-66, 2012.

[23] Peng Wang, Yuming Zhou, and Baowen Xu. Matching large ontologies based on reduction anchors. In Proc. 22nd International Joint Conference on Artificial Intelligence (IJCAI), Barcelona (ES), pages 2343-2348, 2011.

[24] Peigang $\mathrm{Xu}$, Haijun Tao, Tianyi Zang, and Yadong Wang. Alignment results of SOBOM for OAEI 2009. In Proc. 4th ISWC workshop on ontology matching (OM), Chantilly (VA US), pages 216-223, 2009.

[25] Katrin Zaiss, Stefan Conrad, and Sven Vater. A benchmark for testing instance-based ontology matching methods. In Proc. EKAW 2010 Posters and Demos, 2010. 


\begin{tabular}{|c|c|c|c|c|c|c|c|c|c|c|c|c|c|c|c|c|c|c|c|c|c|c|c|c|c|c|c|c|c|c|c|}
\hline \multirow{2}{*}{\begin{tabular}{|l|} 
algo \\
test
\end{tabular}} & \multicolumn{3}{|c|}{1} & \multicolumn{3}{|c|}{2} & \multicolumn{3}{|c|}{ o } & \multicolumn{3}{|c|}{4} & & \multicolumn{3}{|c|}{6} & & \multicolumn{4}{|c|}{$\gamma$} & \multicolumn{3}{|c|}{8} & \multicolumn{3}{|c|}{10} \\
\hline & $\mathrm{P}$ & F. & $\mathrm{R}$. & P. & F. & $\mathrm{R}$. & P. & F. & R. & P. & F. & $\mathrm{R}$. & r. & $\mathrm{F}$ & R. & P. & F. & $\mathrm{R}$. & P. & $\overline{\mathrm{F}}$ & $\overline{\mathrm{F}}$ & & $\mathrm{P}$. & $\mathrm{F}$. & $\mathrm{R}$. & P. & $\mathrm{F}$. & R. & P. & F. & R. \\
\hline \multicolumn{32}{|c|}{ Random } \\
\hline 1 & 95 & 5.76 & 3 & 1.0 & .82 & 9 & 9 & .78 & .64 & .97 & .76 & 6.63 & 99 & .84 & .74 & .86 & .78 & 8.72 & .74 & 84.5 & 56.4 & 55 & .90 & .82 & .76 & .52 & .37 & .29 & 95 & & 4.60 \\
\hline 2 & & .75 & .62 & 1.0 & .82 & 0 & 8 & .78 & .64 & .98 & .76 & 6.62 & .99 & .84 & & .86 & .78 & .72 & 73 & 73.5 & 55.4 & & .90 & .82 & .76 & .50 & 37 & .29 & 95 & 74 & 61 \\
\hline 3 & .95 & 5.75 & .61 & 1.0 & .82 & .69 & .98 & .78 & .64 & .97 & .76 & 6.63 & .99 & .84 & .73 & .86 & .79 & 9.72 & .74 & 74.5 & 56.4 & & .91 & .83 & .76 & .51 & .37 & .29 & 95 & .74 & 4.61 \\
\hline 4 & .95 & 5.75 & .62 & 1.0 & .82 & .69 & .98 & .78 & .64 & .97 & .76 & 6.62 & .99 & .84 & .73 & 85 & .77 & 7.71 & .74 & 74.5 & 66.4 & & .91 & .83 & .77 & .51 & .37 & .29 & 95 & 74 & 4.60 \\
\hline 5 & .96 & .76 & .62 & 1.0 & .82 & .69 & .98 & .78 & .64 & .98 & .76 & 6.62 & .99 & .84 & .73 & 36 & .78 & 8.71 & .74 & 74.5 & 56. & & .90 & .82 & .76 & .52 & 37 & .29 & 94 & 73 & 3.60 \\
\hline Avg & .95 & 5.75 & .62 & 1.0 & .82 & .69 & .98 & .78 & .64 & .97 & .76 & 6.62 & .99 & .84 & .73 & .86 & .78 & 8.72 & .74 & 74.5 & 66.4 .45 & & .90 & .82 & .76 & .51 & 37 & .29 & .95 & .74 & 4.60 \\
\hline Ma & .01 & .01 & .02 & .00 & .00 & 01 & .01 & .00 & .00 & .01 & .00 & 01. & .00 & .00 & .01 & .01 & .02 & 2.01 & .01 & 0. & (. & & .01 & .01 & .01 & .02 & .00 & .00 & .01 & .01 & 1.01 \\
\hline & & & & & & & & & & & & & & ontir & auou & & & & & & & & & & & & & & & & \\
\hline 0 & .96 & 5.76 & .63 & 99 & .84 & .72 & 97 & .85 & .75 & .95 & .78 & .66 & $\mid .98$ & .84 & .73 & \begin{tabular}{|l|l}
.83 \\
\end{tabular} & .76 & 6.70 & .74 & 74.6 & 50.5 & 51 & .93 & .84 & .78 & .55 & 40 & .32 & .99 & .87 & 7.78 \\
\hline 1 & 5 & 5.76 & 3 & 1.0 & .82 & 69 & .99 & .78 & .65 & .97 & .77 & 7.64 & 99 & .85 & .74 & 85 & .78 & 8.72 & .74 & 84.5 & 66. & 15 & .88 & .81 & .75 & .51 & 37 & .30 & .94 & .73 & 3.60 \\
\hline 2 & 7 & 7.78 & .65 & 1.0 & .81 & .68 & 8 & .77 & .64 & .97 & .76 & 6.62 & .99 & .85 & .74 & .86 & .78 & 8.72 & .74 & 74.5 & 56.4 & & .91 & .83 & .77 & .52 & .37 & .29 & .94 & .74 & 4.61 \\
\hline 3 & .95 & 5.75 & .62 & 1.0 & .82 & .69 & .98 & .77 & .63 & .97 & .75 & 5.61 & .99 & .83 & & 36 & .79 & 9.72 & .74 & 74.5 & 55.4 & 14 & .91 & .83 & .76 & .50 & .36 & .29 & 93 & .74 & 4.61 \\
\hline 4 & .96 & 5.75 & .62 & 1.0 & .82 & .69 & .98 & .78 & .64 & .98 & .77 & 7.64 & .99 & .85 & .75 & .88 & .79 & 9.71 & .74 & 74.5 & 56. & & .89 & .82 & .76 & .52 & .38 & .30 & 95 & .74 & 4.60 \\
\hline 5 & .92 & 2.74 & .62 & 1.0 & .80 & .67 & .98 & .77 & .63 & .98 & .76 & 6.63 & .99 & .83 & .72 & .86 & .78 & 8.71 & .74 & 74.5 & 56. & & .88 & .81 & .74 & .51 & .37 & .29 & 93 & .73 & 3.60 \\
\hline & .95 & 5.76 & .63 & 1.0 & .81 & .68 & .98 & .77 & .64 & .97 & .76 & 6.63 & .99 & .84 & .73 & .86 & .78 & 8.72 & .74 & 74.5 & 56. & & .89 & .82 & .76 & .51 & .37 & .29 & 94 & .74 & 4.60 \\
\hline $\mathrm{M}_{\mathrm{c}}$ & .05 & 5.04 & .03 & .00 & .02 & .02 & .01 & .01 & .02 & .01 & .02 & 2.03 & .00 & .02 & .03 & 03 & .01 & 1.01 & .00 & 0 & (1) & 2 & .03 & .02 & .03 & 02 & 02 & .01 & .02 & 01 & 1.01 \\
\hline & & & & & & & & & & & & & & Diffic & ult5 & & & & & & & & & & & & & & & & \\
\hline 1 & .96 & .63 & .46 & 0 & .73 & 58 & 97 & .68 & .52 & .98 & 67 & .51 & 99 & .76 & .61 & .86 & .76 & 6.68 & 65 & 35.4 & 41.3 & & 84 & 71 & .62 & .39 & 26 & .2 & 88 & 60 & .46 \\
\hline & & .63 & 7 & & .74 & & & .68 & .52 & 98 & .66 & 6.50 & .99 & .76 & 62 & 6 & .76 & 68 & 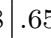 & 4. 55 & 41. & & 34 & 72 & 63 & 10 & 26 & 0 & 39 & 61 & 1.46 \\
\hline 3 & 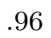 & . 61 & .45 & 1.0 & .73 & 8 & 7 & .67 & .52 & .98 & .66 & 6.50 & .99 & .76 & .62 & .86 & .75 & 5.67 & .66 & 4. 6. & 3. & & .84 & .72 & .63 & .39 & .26 & .20 & 91 & 61 & 1.46 \\
\hline 4 & .96 & .61 & .45 & 1.0 & .74 & .59 & .98 & .68 & .52 & .98 & .66 & 6.50 & & .76 & .62 & .85 & .74 & 4.66 & .65 & 55.4 & 41. & & .84 & .72 & .63 & .40 & .26 & .20 & 87 & .60 & 0.46 \\
\hline & .96 & 63.63 & .46 & 1.0 & .73 & 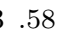 & .98 & .68 & .52 & .98 & .67 & 7.51 & .99 & .76 & .62 & .85 & .75 & 5.66 & .65 & 4. & 41.3 & & .85 & .72 & .63 & 38 & .26 & .19 & 90 & .61 & 1.46 \\
\hline & б & . 62 & .46 & 1.0 & .73 & .58 & .98 & .68 & .52 & .98 & .66 & 6.50 & .99 & .76 & .62 & .86 & .75 & 5.67 & .65 & 4. 55 & 3. & & .84 & .72 & .63 & .39 & .26 & .20 & 89 & 61 & 1.46 \\
\hline Mar & .00 & .02 & .02 & .00 & .01 & .01 & .01 & .01 & .00 & .00 & .01 & 1.01 & .00 & .00 & .01 & .01 & .02 & .02 & .01 & 01. & ס. & & .01 & .01 & .01 & .02 & .00 & .01 & .04 & & 1.00 \\
\hline & & & & & & & & & & & & & & Diff & cult7 & & & & & & & & & & & & & & & & \\
\hline 1 & & 7.55 & 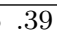 & & .68 & 51 & & .62 & .4 & $\overline{99}$ & .61 & 1.44 & 99 & $\overline{0}$ & .54 & $\overline{87}$ & .70 & 0.59 & .58 & 88.3 & 32. & & $\begin{array}{l}.79 \\
\end{array}$ & .67 & .58 & 31 & .20 & .15 & $\overline{84}$ & .52 & 2.38 \\
\hline 2 & .97 & .55 & 88 & U & .68 & 2 & .97 & .62 & 46 & .99 & .61 & 1.44 & .99 & .70 & .54 & .86 & .69 & 9.58 & .58 & 88.3 & 32. & & .79 & .67 & .58 & .31 & 20 & .15 & .89 & .52 & 2.37 \\
\hline 3 & .9 & .54 & .38 & 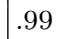 & .68 & 2 & 7 & .62 & .46 & .99 & 61 & 1.44 & .99 & .69 & .54 & 87 & .70 & 0. & 58 & 68.3 & 32. & & .79 & .67 & .58 & 31 & .20 & .15 & 87 & .52 & 2.37 \\
\hline 4 & 6 & 5.55 & .38 & 9 & .68 & 1 & 7 & .62 & .46 & .99 & .61 & 1.44 & .99 & .69 & .53 & .87 & .70 & .59 & .58 & 88.3 & 32.2 & & .79 & .67 & .58 & .31 & .20 &. & 83 & .51 & 1.37 \\
\hline 0 &. & 7.55 & .38 & 9 & .68 & .52 & 1 & .62 & .46 & .99 & .61 & 1.45 & & .70 & & 6 & .69 & 9 & 58 & 88 & 32 . 32 & & .79 & .67 & 8 & 31 & 20 & . & 86 & .52 & 2.37 \\
\hline & 7 & 7.55 & .38 & .99 & .68 & 3 & .97 & .62 & .46 & .99 & .61 & 1.44 & .99 & .70 & .54 & .87 & .70 & 0.59 & .58 & 88 & 32. & & .79 & .67 & .58 & .31 & .20 & . & 86 & 52 & 2.37 \\
\hline & .01 & 1. 01 & .01 & .01 & .00 & & .00 & .00 & .00 & .00 & .00 & 0 & .00 & .01 & .01 & .01 & .01 & 1.01 & .00 & 0 & 0. & & .00 & .00 & .00 & .00 & .00 & .01 & .06 & .01 & 1.01 \\
\hline & & & & & & & & & & & & & & Diffic & ult9 & & & & & & & & & & & & & & & & \\
\hline 1 & .97 & & & .99 & .64 & & 97 & .59 & 42 & .99 & .57 & 7.40 & .99 & .65 & .48 & .88 & .67 & 7.54 & 1.52 & 52.2 & 1. & & .77 & .66 & .57 & .26 & .16 & .11 & .83 & .46 & 6.32 \\
\hline 2 & & 5.50 & 4 & 1.0 & .64 & 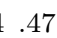 & 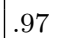 & .59 & 42 & .99 & .57 & 7.40 & .99 & .65 & .48 & 7 & .67 & 7.54 & .53 & 53.2 & 1. & & .75 & .65 & .57 & 26 & .16 & . & 81 & 44 & 4.31 \\
\hline & & 5 & .3 & .99 & .64 & 47 & 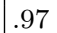 & .59 & 42 & 9 & .57 & 7.40 & .99 & .65 & .48 &.$\varepsilon$ & .67 & 7.54 & .52 & 52.2 & 1. 26 & & .76 & .65 & . & 25 & .16 & & 82 & .46 & 6.32 \\
\hline 4 & .9 & .51 & .34 & .99 & .64 & .47 & .97 & .59 & .42 & .99 & .57 & 7.40 & .99 & .65 & .48 & 38 & .66 & 6.53 & .52 & 52.2 & 1. 26 & & .75 & .64 & .56 & .26 & .16 & .12 & 83 & .46 & 6.31 \\
\hline & .9 & 7.51 & .34 & .99 & .64 & .47 & .97 & .59 & .42 & .99 & .57 & 7.40 & .99 & .65 & .48 & .88 & .67 & 7 & .52 & 52.2 & 1. 26 & 17 & .76 & .65 & 7 & .26 & .16 & .12 & 84 & 46 & 31 \\
\hline & .96 & 5.51 & .34 & .99 & .64 & 47 & .97 & .59 & .42 & .99 & .57 & 7.40 & .99 & .65 & .48 & 38 & .67 & 7.54 & .52 & 62.2 & 26 & & .76 & .65 & & .26 & .16 & . & 83 & 46 & 6.31 \\
\hline Maxvar & .01 & 1. .01 & .01 & .01 & .00 & 0 & .00 & .00 & .00 & .00 & .00 & 00 & .00 & .00 & .00 & .01 & .01 & 1.02 & .01 & 1.0 & C. & & .02 & .02 & 01 & .01 & .00 & .01 & .03 & .02 & 2.01 \\
\hline
\end{tabular}

Table B.10: Bibliography results with 2012 test set ( $\mathrm{P}=$ precision, $\mathrm{F}=\mathrm{F}$-measure, $\mathrm{R}=$ Recall). 


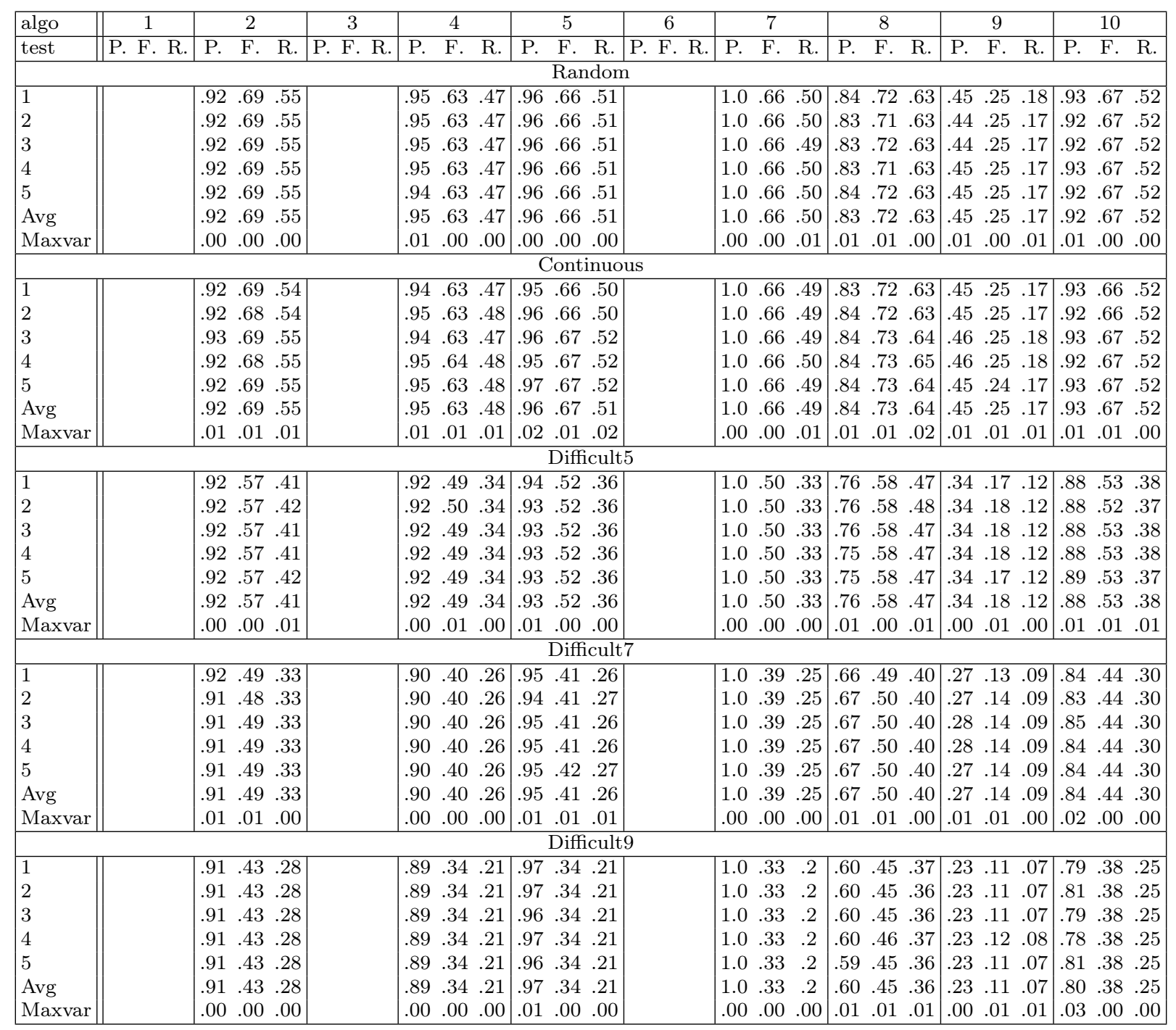

Table B.11: Photography results with 2012 test set ( $\mathrm{P}=$ precision, $\mathrm{F}=\mathrm{F}$-measure, $\mathrm{R}=$ Recall). 


\begin{tabular}{|c|c|c|c|c|c|c|c|c|c|c|c|c|c|c|c|c|c|c|c|c|c|c|c|c|c|c|c|c|c|c|}
\hline \multirow{2}{*}{\begin{tabular}{|l|} 
algo \\
test
\end{tabular}} & \multicolumn{3}{|c|}{1} & \multicolumn{3}{|c|}{2} & \multicolumn{3}{|c|}{3} & \multicolumn{3}{|c|}{4} & \multicolumn{3}{|c|}{5} & \multicolumn{3}{|c|}{6} & \multicolumn{3}{|c|}{7} & \multicolumn{3}{|c|}{8} & \multicolumn{3}{|c|}{9} & \multicolumn{3}{|c|}{10} \\
\hline & P. & $\mathrm{F}$ & R. & $\mathrm{P}$. & F. & $\mathrm{R}$. & P. & $\mathrm{F}$ & R. & P. & $\mathrm{F}$ & R. & & $\mathrm{F}$ & & P. & 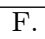 & R. & $\mathrm{P}$ & $\mathrm{F}$ & R. & P. & $\mathrm{F}$ & R. & P. & $\mathrm{F}$ & $\mathrm{R}$. & & $\mathrm{F}$. & R. \\
\hline \multicolumn{31}{|c|}{ Random } \\
\hline 1 & 92 & .68 & .54 & .96 & .73 & 3.58 & 89 & 9.67 & .54 & .77 & .60 & .49 & .85 & .53. & & .92 & .67 & .52 & .99 & .66 & .50 & 82 & .74 & 1.67 & 21 & 12 & .08 & 92 & .73 & 61 \\
\hline 2 & 2 & .68 & .54 & 96 & .72 & .58 & 89 & 9.67 & .54 & .78 & .60 & .49 & .85 & 3. & .38 & .90 & .67 & .54 & .99 & .66 & .50 & 82 & .74 & .67 & .21 & .12 & .08 & .92 & .73 & .61 \\
\hline & & .68 & .54 & 7 & .73 & 3.58 & 90 & .68 & .54 & .78 & .60 & .49 & & 3. & 39 & .90 & .67 & .53 & .99 & 66 & .50 & 82 & .74 & .67 & 21 & .12 & , & 92 & .73 & .01 \\
\hline 4 & .92 & .68 & .54 & 97 & .73 & .58 & 89 & .67 & .54 & .77 & .60 & .49 & .85 & .53 .3 & & .91 & .67 & .53 & .99 & .66 & .50 & 32 & .74 & .67 & 21 & .12 & .08 & .92 & .73 & .61 \\
\hline 5 & .91 & .68 & .54 & 77 & .73 & .58 & .89 & 9.67 & .54 & .78 & .60 & .49 & .85 & 3. & .38 & .90 & .67 & .53 & 99 & 66 & .50 & 82 & .74 & .67 & .21 & 12 & .08 & 92 & .73 & .60 \\
\hline A & .9 & .68 & .54 & .97 & .73 & .58 & .89 & .67 & .54 & .78 & .60 & .49 & .85 & 3. & & 91 & .67 & .53 & 9 & 66 & .50 & 2 & 74 & .67 & 21 & 12 & 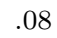 & 92 & 73 & 01 \\
\hline Max & $\mid .01$ & .00 & .00 & .01 & .01 & .00 & .01 & .01 & .00 & .01 & .00 & .00 & ( & co .00. & .01 & .02 & .00 & .02 & .00 & .00 & .00 & .00 & .00 & .00 & .00 & .00 & .00 & .00 & .00 & .01 \\
\hline \multicolumn{31}{|c|}{ Continuous } \\
\hline 1 & 2 & .68 & & 7 & .73 & & 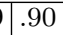 & .67 & .54 & 78 & .60 & .49 & .85 & .52 .3 & .38 & .90 & .67 & 53 & 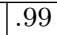 & 66 & 50 & 32 & 74 & 1.01 & 21 & 12 & . & 92 & 73 & \\
\hline 2 & .9 & .68 & .54 & .97 & .73 & 9 & 39 & 9.67 & .54 & .78 & .61 & .50 & .86. & 3. 53. & 39 & .91 & .67 & 3 & 9 & .66 & .50 & 82 & .74 & .67 & 21 & 12 & 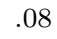 & 2 & .73 & .61 \\
\hline & .92 & .68 & 44 & .97 & .73 & 58 & 0 & .68 & .54 & .79 & .61 & .50 & 85. & .3. & & .91 & .67 & 3 & .99 & .66 & .50 & 2 & .74 & .67 & .21 & 12 & 8 & 92 & .73 & .61 \\
\hline 4 & .92 & .68 & 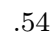 & 96 & .72 & 58 & .89 & .67 & .54 & .77 & .60 & .49 & .85 & 3. & 38 & .91 & .67 & 4 & 9 & .66 & .50 & 82 & .74 & .67 & 21 & 12 & .08 & .92 & .73 & .61 \\
\hline 5 & 1 & .68 & .54 & .97 & .73 & .59 & .89 & 9.67 & .54 & .78 & .61 & .50 & .85 & .53 .3 & & .94 & .67 & .52 & .99 & .66 & .50 & 2 & .74 & .67 & .21 & .12 & 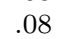 & .92 & .73 & .60 \\
\hline & .92 & .68 & .5 & .97 & .73 & .59 & .89 & 9.67 & .54 & .78 & .61 & .50 & .85 & .3. & & .91 & .67 & .53 & .99 & .66 & .50 & 82 & .74 & .67 & 21 & .12 & 8 & .92 & .73 & .61 \\
\hline & .01 & .00 & .00 & .01 & .01 & .01 & .01 & 1.01 & .00 & .02 & .01 & .01 & & (. 01. & .01 & .04 & .00 & .02 & .00 & .00 & .00 & .00 & .00 & .00 & .00 & .00 & .00 & .00 & .00 & .01 \\
\hline \multicolumn{31}{|c|}{ Difficult5 } \\
\hline 1 & .92 & .54 & .38 & .95 & .60 & $\overline{44}$ & .82 & .53 & .39 & .71 & .49 & .38 & .83. & .46. & .31 & .87 & .54 & 39 & 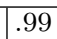 & .50 & .34 & .76 & .63 & .53 & .15 & .08 & .06 & 86 & .61 & .47 \\
\hline & & 53 & 8 & 5 & .60 & & 2 & 2.53 & 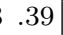 & .71 & .49 & 38 & .83. & .3. & & .89 & .54 & 39 & 9 & .50 & .34 & 76 & .63 & .53 & 16 & .08 & 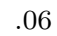 & 86 & .61 & .48 \\
\hline & .91 & .53 & .38 & .95 & .60 & 4 & 32 & 2.53 & 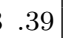 & .71 & .49 & .38 & .83 & 3. & & .88 & .54 & 39 & .99 & .50 & .34 & 76 & .63 & .53 & 15 & .08 & 6 & 86 & .61 & .47 \\
\hline & .01 & .01 & .00 & .00 & .00 & U & .00 & 00 & .00 & .00 & .00 & .00 & ( & . 00.00. & .00 & .02 & .00 & .00 & .00 & .00 & 00 & .00 & .00 & .00 & 01 & .00 & 0 & .00 & .00 & 01 \\
\hline \multicolumn{31}{|c|}{ Difficult7 } \\
\hline 1 & & .45 & & 93 & 52 & & ర & P. & . & .67 & 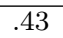 & . & .82. & .40 .2 & & .85 & .46 & . & .99 & 40 & . & 71 & .55 & .45 & 12 & .06 & 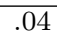 & .82 & .53 & .40 \\
\hline 2 & .9 & .45 & .29 & .94 & .52 & 6 & 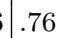 & 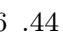 & .31 & .67 & .43 & .31 & .82 & . 2. & .26 & .88 & .45 & .31 & 9 & .40 & .25 & 70 & .55 & .45 & 2 & .07 & . & .82 & .53 & .39 \\
\hline & .9 & .45 & .2 & .93 & .52 & 2 & .76 & 6.44 & . & .67 & .43 & .31 & .82 & . 20. & .26 & .86 & .45 & .31 & .99 & 40 & .2 & 70 & .55 & .45 & 12 & .06 & 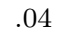 & .82 & .53 & .39 \\
\hline & .00 & .00 & .0 & .01 & .00 & & .01 & 1.01 & 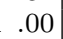 & .00 & 0 & .00 & . & . 00.00. & .00 & .03 & .01 & & 00 & .00 & .00 & 01 & .00 & .00 & O & .01 & & .00 & .00 & \\
\hline \multicolumn{31}{|c|}{ Difficult9 } \\
\hline 1 & .94 & .40 & .25 & .92 & .46 & & 2 & 2.39 & .27 & .64 & .38 & .27 & . & . & 23 & & .40 & .26 & 99 & .33 & .20 & $\overline{3}$ & 3.51 & .42 & 10 & .05 & .04 & \begin{tabular}{|l|}
.79 \\
\end{tabular} & .48 & \\
\hline & & .40 & .25 & .92 & .46 & 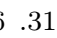 & 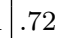 & ) 39 & .27 & .64 & .38 & .27 & S. 81. & 2. 36. & .23 & .86 & .40 & 26 & 9 & .33 & .20 & 63 & 3.51 & .43 & 0 & 5 & 4 & 79 & .48 & 34 \\
\hline & .9 & .40 & .2 & .92 & .46 & .3 & .72 & 2.39 & 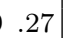 & .64 & .38 & 27 & .81 & 2. & .23 & .87 & .40 & & a & 33 & . & 3 & 3.51 & .43 & 0 & .05 & 4 & .79 & .48 & \\
\hline Maxva & .00 & .00 & .0 & .00 & .00 & & .00 & o0. & .00 & .00 & .00 & .00 & (. & C. 00. & .00 & .01 & .00 & 00 & .00 & .00 & .00 & .00 & .00 & .01 & .00 & .00 & .00 & .00 & .00 & .00 \\
\hline
\end{tabular}

Table B.12: Provenance results with 2012 test set ( $\mathrm{P}=$ precision, $\mathrm{F}=\mathrm{F}$-measure, $\mathrm{R}=$ Recall). 


\begin{tabular}{|c|c|c|c|c|c|c|c|c|c|c|c|c|c|c|c|c|c|c|c|c|c|c|c|c|c|c|c|c|}
\hline \multirow{2}{*}{$\begin{array}{l}\text { algo } \\
\text { test }\end{array}$} & \multirow{2}{*}{$\begin{array}{c}1 \\
\text { F. R. }\end{array}$} & \multicolumn{3}{|c|}{2} & \multicolumn{3}{|c|}{3} & \multicolumn{3}{|c|}{ 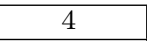 } & \multicolumn{3}{|c|}{5} & \multicolumn{3}{|c|}{6} & \multicolumn{3}{|c|}{7} & \multicolumn{3}{|c|}{0} & \multicolumn{3}{|c|}{9} & \multicolumn{3}{|c|}{10} \\
\hline & & 1. & F. & $\mathrm{R}$. & 1. & F. & R. & P. & F. & R. & P. 1 & F. $\mathrm{R}$ & R. & 1. & F. & $\mathrm{R}$. & . & F. & R. & & F. & R. & 1. & F. & R. & 1. & F. & R. \\
\hline \multicolumn{29}{|c|}{ Random } \\
\hline & & .99 & 5 & .67 & 3 & 79 & .69 & $\overline{0}$ & .70 & .57 & .90. & .67 .5 & 53. & .92 & .77 & .66 & 4 & .64 . & .49 & 3 & .79 & .69 & 4 & .19 & .13 & 7 . & .79 & .07 \\
\hline & & & .80 & .67 & 3 & .79 & .69 & & .70 & .57 & .90. & .67 .5 & & 2 & .77 & 67 & & .64 & .49 & 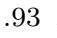 & .79 & & 4 & .19. & .13 & & .79 & .67 \\
\hline & & .99 & .79 & .67 & .93 & 79 & .69 & .90 & .69 & .57 & .90. & .67 .5 & & .92 & .77 & .66 & 4 & .64 & .49 & 93 & .79 & .69 & 3 & .18 & .13 & 97. & 79 & .67 \\
\hline & & & .79 & .67 & .93 & 79 & .69 & .90 & .70 & .57 & .90. & .67 .5 & .53 . & .92 & .77 & .66 & 4 & .64 & .49 & 93 & .79 & .69 & 34 & .19 & .13 & 6 & 79 & .67 \\
\hline & & & .80 & .67 & .93 & .79 & .69 & .89 & .69 & .57 & .90. & .67 .5 & & .92 & .77 & .66 & 4 & .64 . & .49 & 93 & .79 & & 4 & .19 & .13 & 7 . & .79 & 57 \\
\hline & & .99 & .80 & .67 & .93 & 79 & .69 & .90 & .70 & .57 & .90. & .67 .5 & & .92 & .77 & .66 & 94 & .64 . & .49 & 93 & .79 & .69 & 34 & .19 . & .13 & 97. & 79 & .67 \\
\hline & & .00 & .01 & .00 & .00 & .00 & .00 & .01 & .01 & .00 & .00. & C. & . 00 & .00 & .00 & .01 & 00 & .00 . & .00 & .00 & .00 & .00 & 01 & .01. & .00 & 01. & .00 & .00 \\
\hline \multicolumn{29}{|c|}{ Continous } \\
\hline & & .99 & 80 & .67 & 3 & .79 & .69 & .90 & 70 & .57 & .90. & .66 .5 & .52 & .91 & .78 & 68 & 5 & .64 & .49 & 3 & .79 & .69 & 4 & .19 & .13 & 6 . & .78 & (66 \\
\hline & & & 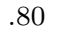 & & & .79 & .69 & 0 & .70 & .57 & .90. & .66 .5 & & 2 & .77 & 66 & 4 & . & .48 & 2 & .78 & & 4 & .19 & .13 & 6 & 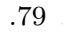 & 67 \\
\hline & & & .80 & .67 & 3 & 79 & .69 & .90 & .70 & .57 & 90. & .66 .5 & & .92 & .78 & .67 & 5 & .64 . & .49 & 3 & .79 & 9 & 34 & .19. & .13 & 6 & 79 & .67 \\
\hline & & .0 & .00 & .00 & .00 & .00 & .00 & .00 & .00 & .00 & .00. & . 00. & .01. & . 01 & .01 & .02 & 01 & .00 & .01 & .01 & .01 & .01 & 00 & .00 & .00 & 00 & & .01 \\
\hline \multicolumn{29}{|c|}{ Difficulty $=.5$} \\
\hline & & & 1 & & & .71 & .58 & & .01 & .46 & 89. & .61 .4 & 46 & .86 & .68 & 7 & 2 & .50 & 4 & 1 & .08 & 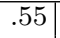 & 4 & .13 & .09 & \pm & 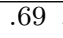 & .55 \\
\hline & & & .52 & .35 & .91 & 71 & .58 & 9 & .61 & .46 & .89. & . 61. & & .87 & .69 & .57 & 2 & .50 & 4 & 2 & .69 & . & 5 & .13 & 8 & 4. & 69 & .55 \\
\hline & & .99 & .52 & $\therefore$ & 1 & .71 & .58 & .89 & .61 & .46 & .89. & .61 .4 & .46 & .87 & .69 & . & 2 & .50 & . & 92 & .69 & . & 25 & .13 & .09 & 4 & 1 & .55 \\
\hline & & ) & .01 & f & 00 & .00 & 00 & .00 & .00 & .00 & .00. & C & .00 & .01 & .01 & .00 & .00 & .00 & ) & 01 & d & 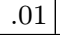 & 01 & .00 & .01 & 00 & & .00 \\
\hline \multicolumn{29}{|c|}{ Difficulty $=.7$} \\
\hline 1 & & .97 & .63 & & .90 & .66 & .52 & .88 & .55 & 40 & .89. & .57 .4 & .42 & .86 & .58 & 44 & 91 & .41 & .26 & 89 & .63 & .48 & 19 & .10 & .06 & 2. & .63 & \\
\hline & & & .63 & & & .66 & .52 & .89 & .55 & .40 & .89 & .58 .4 & & .86 & .58 & 4 & .91 & .41 & .26 & 9 & & . & 9 & .09 & .06 & 2. & 3 & .47 \\
\hline & & .97 & .63 & .46 & .90 & .66 & .52 & 9 & .55 & .40 & .89 & .58 & .43 & .86 & .58 & 4 & 1 & .41 & .26 & 89 & .63 & & 9 & .10 & .06 & 2 & .63 & .48 \\
\hline & & .00 & .00 & .00 & .00 & .00 & .00 & .01 & .00 & .00 & .00. & (. & . 01 & .00 & .00 & 00 & .00 & .00 & .00 & 00 & .00 & .00 & .00 & .01 & .00 & 00 & 00 & . 01 \\
\hline \multicolumn{29}{|c|}{ Difficulty $=.9$} \\
\hline & & & .57 & .40 & .8 & 63 & .4 & & .51 & .36 & .90. & .55 & .40 & .85 & .52 & .3 & 5 & .3 & .22 & & & .4 & 5 & .07 & .0 & .91 & 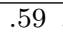 &. \\
\hline & & & .57 & .4 & .89 & .63 & .49 & .89 & .51 & .36 & .90 & .55 & .40 & .86 & .50 & .35 & & .35 & .22 & 5 & .60 & .4 & 5 & .08 & .05 & 1 & .59 & 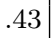 \\
\hline & & 10 & .57 & .41 & .89 & 63 & .49 & .89 & .51 & .36 & .90 & .55 & & .86 & .51 & .36 & .90 & .35 & .22 & 85 & .60 & .47 & .15 & .08 & .05 & .91 & & \\
\hline & & .00 & .00 & .01 & .00 & .00 & .00 & .00 & .00 & .00 & .00 & (. 00. & .00 & .01 & .02 & .02 & .00 & .00 & .00 & .00 & .00 & .01 & .00 & .01 & .00 & .00 & .00 & \\
\hline
\end{tabular}

Table B.13: Finance results with 2012 test set $(\mathrm{P}=$ precision, $\mathrm{F}=\mathrm{F}$-measure, $\mathrm{R}=$ Recall). 